\title{
Applicable and Comparative Research of Compressor Mass Flow Rate and Isentropic Efficiency Empirical Models to Marine Large-Scale Compressor
}

\author{
Haosheng Shen ${ }^{1}$, Chuan Zhang ${ }^{1}$, Jundong Zhang ${ }^{1, *}$, Baicheng Yang ${ }^{2}$ and Baozhu Jia ${ }^{3}$ \\ 1 College of Marine Engineering, Dalian Maritime University, Dalian 116026, China; \\ shenhaosheng@dlmu.edu.cn (H.S.); zc1016@dlmu.edu.cn (C.Z.) \\ 2 College of Navigation, Dalian Maritime University, Dalian 116026, China; yangbaicheng@dlmu.edu.cn \\ 3 Marine College, Guangdong Ocean University, Zhanjiang 524088, China; skysky@dlmu.edu.cn \\ * Correspondence: zhjundong@dlmu.edu.cn; Tel.: +86-134-7894-8607
}

Received: 22 October 2019; Accepted: 14 December 2019; Published: 20 December 2019

\begin{abstract}
A compressor is an indispensable component of marine large two-stroke diesel engines. For this type of engine, the compressor mass flow rate and isentropic efficiency empirical models are preferred for both the working cycle dynamic simulation research and the design and testing of control and diagnostics algorithms due to their compact and simple structures, and satisfactory prediction accuracy. Due to absence of comprehensive applicable and comparative research on compressor mass flow rate and isentropic efficiency empirical models for large-scale marine compressors in the literature, two marine compressors with different size, flow rate range, and speed range were selected as research objects in this paper, and a relevant study was conducted to compare and analyze the prediction ability of several classical models, and some recently proposed compressor mass flow rate and isentropic efficiency empirical models. The range of this comparative study includes the prediction accuracy in the design operating area and the extrapolation ability in off-design operating areas. Based on the obtained research results, several guidelines are summarized, which can be followed when developing compressor mathematical models, especially for marine applications. In addition, several research interests are discussed and presented, which can be further studied in the future.
\end{abstract}

Keywords: marine compressor; empirical model; mass flow rate; isentropic efficiency; comparative research

\section{Introduction}

Due to the vital role of the turbocharger in improving power density, lowering fuel consumption, and reducing emissions for diesel engines, researchers have conducted extensive theoretical and experimental studies to exploit its capability further. In the field of automotive engines, several new types of turbocharging systems have emerged, such as electrically assisted turbocharging systems (E-Turbo), variable geometry turbochargers (VGTs), and variable geometry compressors (VGCs). It was revealed by many pieces of research that, compared with traditional turbocharging technology, these advanced technologies can not only effectively improve turbocharger performance at low loading conditions but also substantially extend the operating range [1,2]. Nowadays, large marine two-stroke diesel engines are generally equipped with a turbocharging system but they still lag behind automotive engines to some extent. Nevertheless, the turbocharging system substantially contributes to an improvement in power, economic and emission performance for marine diesel engines, due to the fact that they can effectively increase the density of scavenging air, which not only contributes to the 
complete burning of injected fuel but also allows more fuel to be injected into the combustion chamber in a single working cycle [3].

Due to the important role of turbochargers for both automotive and marine diesel engines, it is indispensable to develop corresponding mathematical models with satisfactory predictive accuracy for not only the working cycle dynamic simulation research of turbocharged diesel engines but also the design and testing of control and fault diagnosis algorithms. Therefore, researchers have conducted extensive studies on modeling the methodologies of turbochargers. The modeling methodologies in the existing literature exhibit different levels of complexity, accuracy, and computational requirements, which can be roughly classified into the look-up table method, the curve fitting method, the neural network method, and the theoretical modeling method [4]. It should be noted that most research focuses on small-scale automotive turbochargers, and relevant research on large-scale marine turbochargers are scarce. The reason is that the large size and high flow range of marine turbochargers requires special and expensive experimental equipment, making it very costly to carry out relevant experimental studies; in addition, the manufacturers of marine turbochargers rarely provide turbocharger performance maps, which makes it very difficult for model development and tuning. Nevertheless, some relevant studies on large-scale marine turbochargers, especially for compressors, can be found in the literature. In [5], the compressor mass flow rate was expressed as a polynomial function of rotational speed and pressure ratio. Karlson [6] utilized the exponential function to fit the relationship between the dimensionless flow coefficient and the head coefficient for a compressor, and the isentropic efficiency was expressed as a polynomial function of the mass flow rate and rotational speed. Hansen et al. [7] used a two-dimensional fourth-order polynomial to model the mass flow rate and isentropic efficiency of a compressor. Sun et al. [8] developed a compressor mathematical model that can predict the surging characteristics and proposed a method to determine the characteristics curve of a compressor under unstable working conditions. Tu and Chen [9] attempted to model compressor mass flow rates based on the Kriging algorithm. Wang et al. [10] developed a theoretical model for a marine centrifugal compressor based on thermodynamic laws. In [11], a marine turbocharger model based on the average line method was proposed, the highlight of which was that the model could be tuned with a relatively small amount of measured data.

By analyzing the above-mentioned researches, it can be found that most modeling methodologies of marine compressors for both the mass flow rate and isentropic efficiency fall into the curve fitting method category. This is due to the fact that relative to their lookup table counterparts, stronger physical fundamentals can be embedded into the models developed with this method. In addition, they are much more computationally efficient than those developed with theoretical modeling methods. As a result, the curve fitting method can yield time-efficient mathematical models with compact and simple structures, which can not only satisfy the requirements of control- and/or diagnostic-oriented dynamic simulations, but also possibly apply to design, analysis, and performance simulation [12]. It should be noted that the models developed with the curve fitting method are often referred to as empirical, semi-empirical, or mean value models in the literature.

By extensive literature survey, we have not found any paper providing a comprehensive applicable and comparative study of compressor mass flow rates and empirical isentropic efficiency models to a large-scale marine compressor. Two similar papers can be found in the literature. However, their research object was the centrifugal compressor for jet engines and refrigeration systems, respectively, and in addition, they only focused on the design operating area [12,13]. To fill in this gap, two large-scale marine compressors with different sizes, flow rates, and speed ranges were selected as the research object in this paper, and a comprehensive applicable and comparative analysis was conducted to compare and analyze the predictive ability of several classical models, and several recently proposed compressor mass flow rate and empirical isentropic efficiency models. The comparative range includes both the design and off-design operating area.

The rest of the paper is organized as follows: The characteristic of compressor performance map and the definitions of several relevant dimensionless numbers is first introduced in Section 2. Then, 
various compressor mass flow rate and isentropic efficiency models are described briefly in Section 3. After that, the main technical parameters of two marine compressors, comparative methodology, and error evaluation criteria are presented in Section 4. Results of the comparisons and analysis results of mass flow rate and isentropic efficiency models are given in Sections 5 and 6, respectively. Section 7 summarizes the comparative and analysis results and discusses some limitations of existing empirical compressor models and feasible solutions. The last section of this paper is dedicated to conclusions and several guidelines are summarized, which can be followed when selecting or developing models for large-scale marine compressors.

\section{Compressor Performance Map and Dimensionless Numbers}

\subsection{Compressor Performance Map}

The working characteristic of a compressor is usually represented by its performance map, as shown in Figure 1, with mass (or volume) flow rates and pressure ratios as the horizontal and vertical coordinates, respectively. In the performance map, measured data points with the same rotational speed are connected, forming the iso-speed lines, and those with the same isentropic efficiency are connected forming the iso-efficiency lines. Essentially, the compressor mass flow rate and isentropic efficiency empirical models can be considered as a mathematical description of the performance $\operatorname{map}[12]$.

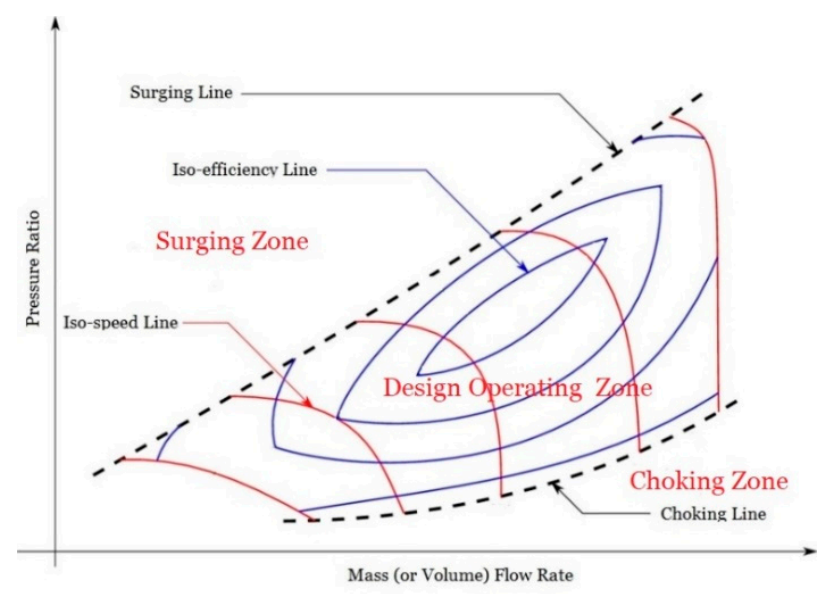

Figure 1. Compressor performance map.

To cover different operating environments, corrected quantities including the corrected rotational speed $N_{\text {corr }}$ and corrected mass flow rate $\dot{m}_{\text {corr }}$ are usually utilized in the performance map with their definitions as follows

$$
\begin{aligned}
& N_{\text {corr }}=N \frac{1}{\sqrt{T_{\text {in }} / T_{\text {ref }}}} \\
& \dot{m}_{\text {corr }}=\dot{m} \frac{\sqrt{T_{\text {in }} / T_{\text {ref }}}}{p_{\text {in }} / p_{\text {ref }}}
\end{aligned}
$$

where $T_{\text {ref }}$ and $p_{\text {ref }}$ are the reference temperature and pressure, respectively, $T_{\text {in }}$ and $p_{\text {in }}$ are the temperature and pressure at the compressor inlet, respectively. The derivation of Equations (1) and (2) is based on the dimensional analysis of the compression process in a compressor [14].

For a compressor, its whole operating area is normally divided into three zones, as shown in Figure 1, i.e., design operating zone, surging zone, and choking zone. In the design operating zone, the compressor is able to work stably and achieve high efficiency. In the surging zone, the momentum of airflow cannot stably overcome the adverse pressure gradient across the compressor, and reverse flow occurs periodically, resulting in a mild flutter to a wildly fluctuating boost, and the resultant noise 
known as "surging" $[12,15]$. To prevent premature turbo failure, the compressor should avoid working in this zone continuously. The curve connecting the surging point on each iso-speed line is referred to as a surging line. When entering the choking zone, the flow reaches a state of sonic velocity, and the mass flow rate will no longer increase despite the decrease in pressure ratio, which is characterized by a steeply descending iso-speed line at the lower right side of the performance map, as shown in Figure 1. Similar to the surging line, the curve connecting the choking point on each iso-speed line is referred to as the choking line.

As shown in Figure 1, most of the compressor performance maps provided by turbocharger manufacturers only contain discrete measured data points in the design operating zone; however, for marine compressors, its actual rotational speed may be lower than the lowest rotational speed showed in the performance map, or higher than the highest one, and its pressure ratio may be lower than one under certain working conditions, such as main engine low-load running, activation of the auxiliary blower, and ship maneuvering [16]. Therefore, it is required that the developed compressor model is capable of extrapolating to these off-design operating zones accurately and reasonably. In addition, since the performance map only provides several discrete iso-speed and iso-efficiency lines, the developed model is also required to interpolate within the unknown zones between these discrete lines with sufficient accuracy. Based on the above analysis, the range of applicable and comparative analysis conducted in this paper includes both the predictive accuracy in the design operating area and the extrapolation ability in the off-design operating areas. The off-design operating areas include the area with rotational speed lower than the lowest one available in the performance map, the area with rotational speed higher than the highest one, and the area to the right of the curve that connects the measured operating point of each iso-speed line with highest flow, which are termed LS, HS and LP area in this paper for convenience of expression, respectively

It should be noted that by observing and comparing the performance map of small-scale automotive and large-scale marine compressors, it is known that the latter has a narrower shape, and its choking and surging lines are much closer than the former [16]. Due to these differences, some compressor mass flow rates and isentropic efficiency empirical models that are well suited to small-scale automotive compressors may not be suitable for large-scale marine compressors.

\subsection{Dimensionless Numbers}

When developing compressor mass flow rate and isentropic efficiency empirical models, several dimensionless numbers are often utilized mainly including head coefficient $\Psi$, flow coefficient $\phi$, and Mach number $M$, and their definitions are as follows [17]

$$
\begin{gathered}
\Psi=\frac{c_{\mathrm{p}} T_{\text {in }}\left(\Pi^{\frac{k-1}{k}}-1\right)}{0.5 \cdot U^{2}} \\
\phi=\frac{\dot{m}}{\frac{\pi}{4} \rho d^{2} U} \\
M=\frac{U}{\sqrt{k R T_{\text {in }}}} \\
U=\frac{\pi d N}{60}
\end{gathered}
$$

where $c_{p}, k, R$ and $\rho$ are the constant pressure specific heat, specific heat ratio, gas constant, and density of air, respectively; $d$ and $U$ is the impeller outlet diameter and the blade tip speed, respectively.

By observing Equations (3)-(6), it is found that most of the physical quantities involved in the compression process of a compressor are covered by these dimensionless numbers, which greatly facilitates the model development process. Essentially, the theoretical foundation of many compressor 
empirical models existing in the literature is the identification of the functional relationship among these dimensionless numbers [18-20].

\section{Compressor Mathematical Models}

Researchers around the world have proposed various types of compressor modeling methodologies with different levels of complexity and accuracy, which can be roughly classified into four categories as following:

- Look-up table method. This method utilizes the measured data points available in the compressor performance map to establish two-dimensional interpolation mesh, as shown in Figure 2, with which the mass flow rate and isentropic efficiency can be calculated at given rotational speed and pressure ratio conditions by using an appropriate interpolation algorithm. Since it is easy to realize, this method has been adopted in many numerical simulation studies [21,22]. Nevertheless, it should be noted that because the standard interpolation algorithm is not continuously differentiable, simulation results with obvious discontinuous points may be obtained. In addition, the extrapolation results obtained with this method are not reliable due to lacking strong physical fundamentals [23].

- Neural network method. Due to the strong nonlinear mapping and generalization ability of the neural network, many researchers have attempted to apply this method to the prediction of the working characteristics of a compressor, and satisfactory results are attained [24,25]. It was pointed out by Gholamrezaei and Ghorbanian [25] that BP (Back-Propagation) neural network with the Levenberg-Marquardt algorithm can achieve satisfactory prediction accuracy. The difficulty of this method lies in how to determine the structure of the neural network appropriately. The under-fitting phenomenon may occur if the structure is too simple and the quantity of nodes is too less; on the other hand, although the prediction capability of the neural network may improve to some extent by increasing its structural complexity and the number of nodes, its generalization ability may degrade, i.e., over-fitting phenomenon may occur;

- Curve fitting method (empirical, semi-empirical, or mean value models). This method is widely used for predicting the working characteristics of compressors, mainly owing to its simpler and much more compact model structure than their theoretical counterparts, as well as their satisfactory predictive and extrapolation ability [3,6,16-20,26-29]. The curve fitting method can be further divided into two sub-categories: Some studies directly fit the relationship between the rotational speed, mass flow rate, pressure ratio, and isentropic efficiency with appropriate functions; while some researchers utilize suitable functions to fit the relationship between different dimensionless numbers derived from measured quantities. It should be noted that the difficulty of this method lies in how to select appropriate fitting functions and the determination of initial guesses of model parameters.

- Theoretical method. The parameterization of compressor models developed with the above mentioned three methods strongly depends on the performance map; thus, their extrapolation ability is limited to some extent. Aiming at this problem, some researchers have attempted to develop theoretical models to predict the working characteristics of compressors without the help of performance maps. These theoretical models can be divided into 3-D, 2-D, and 1-D models in order of decreasing complexity [2,11,30-32]. Due to the large computational requirements, the 3-D and 2-D theoretical models are not suitable for control- and diagnostic-oriented dynamic simulations. Moreover, detailed compressor geometric dimension information is always needed. Compared with 3-D and 2-D models, the structure of 1-D theoretical models is relatively simpler and more compact, and it only requires basic geometric information. For developing 1-D theoretical models, it was first assumed that the compression process of a compressor is the isentropic compression process of compressible fluid, based on which the working characteristics of a compressor can be preliminarily estimated with the Euler equation, continuity equation, and energy conservation 
equation. Then, various types of losses (friction loss, incidence loss, shock loss, slip, wake loss, etc.) occurring during the actual compression process are modeled with the help of well-known empirical and semi-empirical formulas. Finally, the working characteristics of a compressor can be predicted by combing the results of the above two steps. It should be noted that although the theoretical foundation of 1-D theoretical models is the physical description of the actual compression process, many model parameters still need to be parameterized with the measured data provided in the performance map; in addition, some of the geometric information required by the 1-D theoretical model is still difficult to obtain, especially for large-scale marine compressors.

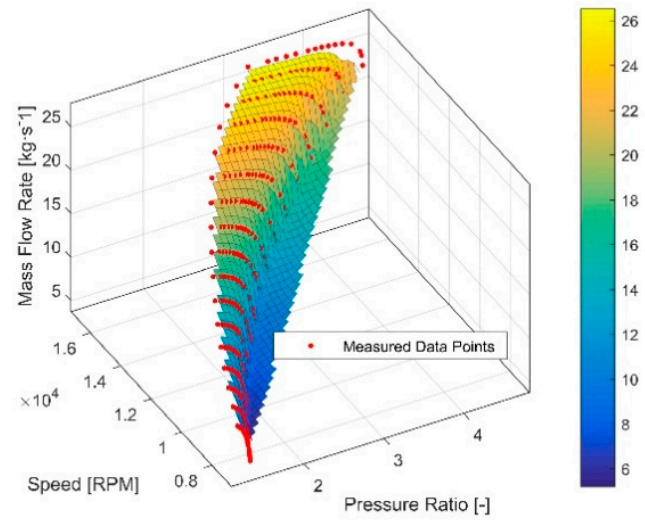

(a)

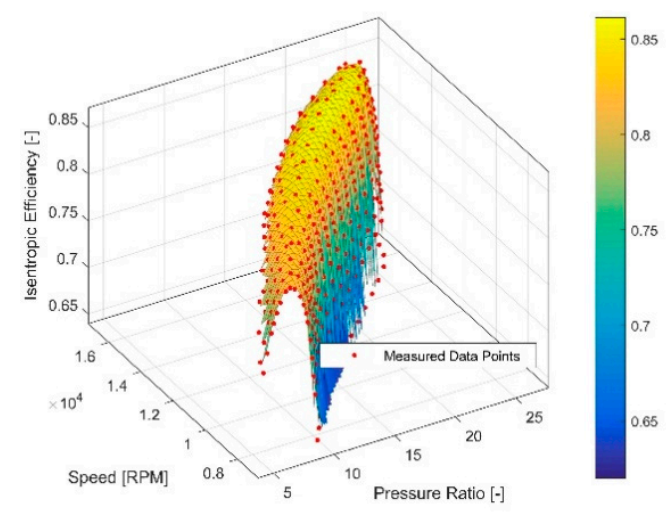

(b)

Figure 2. Two-dimensional interpolation meshes constructed by look-up table method: (a) mass flow rate model; (b) isentropic efficiency model.

Because the ultimate purpose of this paper is to find a compressor model that is most suitable for the modeling of marine two-stroke diesel engine used for the marine engineering simulator, which has a relatively high requirement on both simulation speed and predictive accuracy, so only the empirical models developed with the curve fitting method are compared and analyzed in this paper owing to their simplicity, superior time-efficiency, and satisfactory predictive ability.

\subsection{Compressor Mass Flow Rate Models}

This section will briefly introduce several classical models and recently proposed compressor mass flow rate empirical models. In terms of the selection of the input and output parameters, these models can be divided into two categories with the model structure, as shown in Equations (7) and (8), respectively. Moraal and Kolmanovsky [23] pointed out in their research that the models manifested different strengths and weaknesses in terms of their sensitivity to input errors and model rigidity. Nevertheless, most researchers still select Equation (7) to develop compressor mass flow rate sub-models in the working cycle dynamic simulation model of turbocharged diesel engines, i.e., pressure ratio and rotational speed as the input, and mass flow rate as the output [33,34].

$$
\begin{aligned}
& \dot{m}=f(\Pi, N) \\
& \Pi=f(\dot{m}, N)
\end{aligned}
$$

It should be noted that there are several models that are capable of working in both directions, such as the Leufvén and Llamas Ellipse Model introduced in Section 3.1.7 [3,16,28,29].

\subsubsection{The Guan Cong Model}

Guan et al. [34] proposed a zonal modeling approach for marine compressors, which is referred to as the Guan Cong model in this paper. The basic idea of the Guan Cong model is to divide the whole 
operating area into several zones according to the iso-speed lines available in the performance map, and then utilize Equation (9) to fit the relationship between $\phi, \Psi$, and $M$ in each zone:

$$
\phi=\frac{k_{1}+k_{2} M+k_{3} \Psi+k_{4} M \Psi}{k_{5}+k_{6} M+\Psi}
$$

The six model parameters $k_{1}$ to $k_{6}$ in Equation (9) can be determined through the least square method with the measured data points at the upper and lower boundaries of the iso-speed line of the current zone. Due to lacking lower boundary iso-speed line for the first zone, the model parameters corresponding to the lowest iso-speed line are used when extrapolating into this zone, similarly, for the last zone, the model parameters corresponding to the highest iso-speed line are utilized. Undoubtedly, the extrapolation strategy adopted by the Guan Cong model will inevitably influence its extrapolation accuracy, especially for the HS area, where the compressibility of air changes with compressor rotational speed significantly.

\subsubsection{The Karlson Exponential Model}

When developing the mass flow rate model for the TCA55 marine compressor, Karlson [6] proposed two types of exponential models with a different structure to fit the relationship between $\phi$ and $\Psi$, which are referred to as the Karlson-I and Karlson-II models, respectively. Equation (10) presents the Karlson-I model [6]

$$
\phi=a+\left(1-e^{\Psi^{c}+b}\right)
$$

where $a, b$, and $c$ are all functions of the Mach number.

Karlson-II model is defined as follows [6]

$$
\phi=\alpha\left(1-e^{\beta\left(\frac{\Psi}{\Psi_{\mathrm{th}}}-1\right)}\right)
$$

where, $\alpha, \beta$, and $\Psi_{\text {th }}$ are also expressed as polynomial functions of Mach number, similar to the Karlson-I model.

\subsubsection{The Malkhede Model}

When developing the MVEM (mean value engine model) for a marine turbocharged diesel engine, Malkhede et al. [5] directly adopted the polynomial function as shown in Equation (12) to calculate the mass flow rate of the compressor at given rotational speed and pressure ratio conditions:

$$
\dot{m}=a_{1}+a_{2} N+a_{3} N^{2}+a_{4} N^{3}+\frac{a_{5}}{\Pi}+\frac{a_{6}}{\Pi^{2}}+\frac{a_{7}}{\Pi^{3}}+a_{8} \frac{N}{\Pi}+a_{9} \frac{N}{\Pi^{2}}+a_{10} \frac{N^{2}}{\Pi}
$$

\subsubsection{The Kolmanovsky Model}

Similar to the Karlson model, the Kolmanovsky model utilizes the exponential function to describe the relationship between the flow coefficient and the pressure ratio [23].

Polynomial functions, as shown in Equations (13) and (14) are used to describe the changing trend of flow coefficient and pressure ratio with compressor rotational speed at the surging line:

$$
\begin{aligned}
& \phi_{\text {sur }}=k_{1} N+k_{2} N^{2} \\
& \Pi_{\text {sur }}=k_{3}+k_{4} \phi_{\text {sur }}^{2}
\end{aligned}
$$


For the area to the right side of the curve represented by Equations (13) and (14), the relationship between $\phi$ and $\Pi$ can be described with the exponential function as follows:

$$
\begin{gathered}
\frac{\phi}{\phi_{\text {sur }}}=1+\alpha\left(1-e^{k_{5}\left(\frac{\Pi}{\Pi_{\text {sur }}}-1\right)}\right) \\
\alpha=k_{6} e^{-k_{7} N}
\end{gathered}
$$

For the left side, the relationship can be described with the following linear function:

$$
\frac{\phi}{\phi_{\mathrm{sur}}}=1-\alpha k_{5}\left(\frac{\Pi}{\Pi_{\mathrm{sur}}}-1\right)
$$

\subsubsection{The Müller-I Model}

Müller-I model was developed by appropriately simplifying the compressor 1-D theoretical model developed by Müller et al. [35]. For the Müller-I model, the specific isentropic enthalpy change $\Delta h_{\text {is }}$ is expressed as a function of compressor blade tip speed $U$ and mass flow rate $\dot{m}$

$$
\Delta h_{\mathrm{is}}=U^{2}\left(A\left(\frac{\dot{m}}{U}\right)^{2}+B\left(\frac{\dot{m}}{U}\right)+C\right)
$$

where $A, B$, and $C$ are functions of $U$.

In turn, the pressure ratio can be derived from the $\Delta h_{\text {is }}$ obtained by Equation (18) based on the isentropic compression process as follows:

$$
\Pi=\left(\frac{\Delta h_{\mathrm{is}}}{c_{\mathrm{p}} T_{\mathrm{in}}}+1\right)^{\frac{k}{k-1}}
$$

\subsubsection{The Müller-II Model}

Besides the Müller-I model, Müller et al. [35] also proposed another compressor mass flow rate model with simpler structure as shown in Equation (20), which is referred to as the Müller-II model in this paper:

$$
\Delta h_{\mathrm{is}}=s_{1} U^{2}+s_{2} \dot{m}^{2}+s_{3} \dot{m}+s_{4}
$$

The theoretical foundation of the Müller-II model is that $\Delta h_{\text {is }}$ it is mainly influenced by $U^{2}$, meanwhile, $\dot{m}$ has relatively less influence on $\Delta h_{\text {is }}$ under low and medium rotational speed conditions, which happens to be a primary operating area for a compressor.

\subsubsection{The Leufvén and Llamas Ellipse Model}

The idea of the Leufvén and Llamas ellipse model is showed graphically in Figure $3[3,16,28,29]$. By using the choking line (ChL) and the zero-slope line (ZSL), the whole operating area is divided into three zones: design operating zone, choking zone, and surging zone. ChL is the curve connecting the choking point of each iso-speed line, and ZSL is a curve that connects to the surging point. The iso-speed line between ZSL and ChL is represented by a super-ellipse with a curvature of CUR and crossing both the choking point $\left(\dot{m}_{\mathrm{ch}}, \Pi_{\mathrm{ch}}\right)$, and surging point $\left(\dot{m}_{\mathrm{zs}}, \Pi_{\mathrm{zs}}\right)$. 


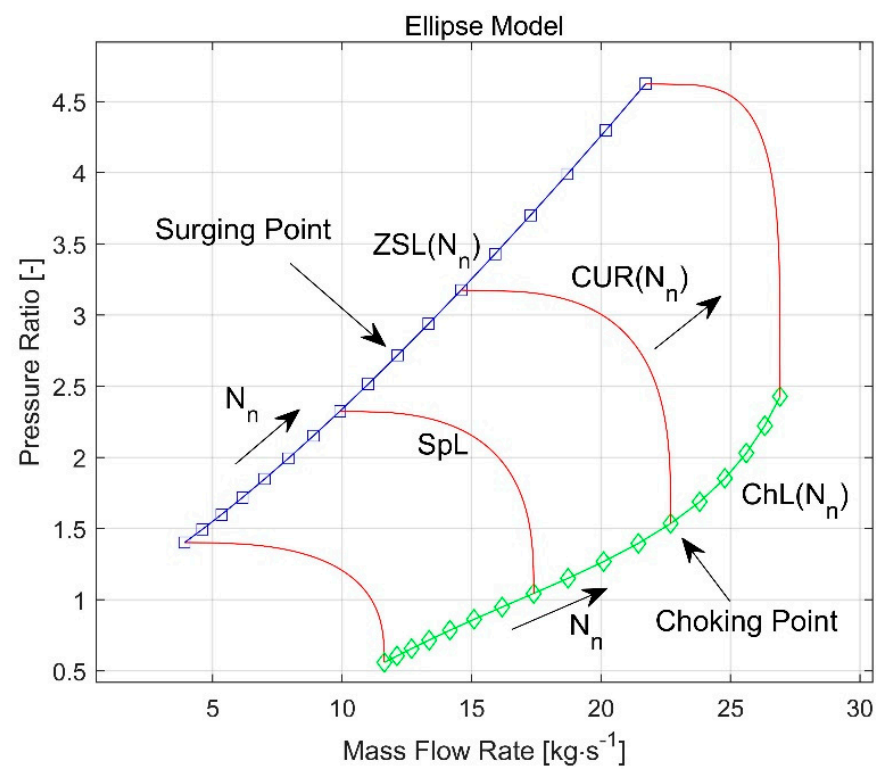

Figure 3. Sketch of the main characteristics of the Leufvén and Llamas ellipse model.

- Design operating zone $\left(\dot{m}_{\mathrm{zs}} \leq \dot{m} \leq \dot{m}_{\mathrm{ch}}\right)$

In the design operating zone, a super-ellipse with a curvature of CUR is used to describe the relationship between $\dot{m}$ and $\Pi$

$$
\left(\frac{\dot{m}-\dot{m}_{\mathrm{zs}}}{\dot{m}_{\mathrm{ch}}-\dot{m}_{\mathrm{zs}}}\right)^{\text {CUR }}+\left(\frac{\Pi-\Pi_{\mathrm{ch}}}{\Pi_{\mathrm{zs}}-\Pi_{\mathrm{ch}}}\right)^{\text {CUR }}=1
$$

where $\dot{m}_{\mathrm{zs}}, \dot{m}_{\mathrm{ch}}, \Pi_{\mathrm{zs}}, \Pi_{\mathrm{ch}}$, and CUR are all functions of normalized compressor rotational speed $N_{\mathrm{n}}$ $\left(N_{\mathrm{n}}=N / N_{\max }\right)$.

- Choking zone $\left(\dot{m}>\dot{m}_{\mathrm{ch}}\right)$

When entering the choking zone, the mass flow rate will no longer change with the pressure ratio. Therefore, a straight line with a slope of infinity and crossing the choking point can be used to describe the changing trend of the iso-speed line in this zone:

$$
\dot{m}=\dot{m}_{\mathrm{ch}}
$$

- Surging zone $\left(0 \leq \dot{m}<\dot{m}_{\mathrm{zs}}\right)$

To reduce the model complexity, it was pointed out by Llamas and Eriksson [16] that a straight line with a slope of $\dot{m}_{\max } / 0.15 \Pi_{\max }$ crossing the zero slope point $\left(\dot{m}_{\mathrm{zs}}, \Pi_{\mathrm{zs}}\right)$ can be used to describe the changing trend of the iso-speed line in this zone:

$$
\dot{m}=\dot{m}_{\mathrm{zs}}-\frac{\dot{m}_{\max }}{0.15 \Pi_{\max }}\left(\Pi-\Pi_{\mathrm{zs}}\right)
$$

Actually, the ellipse model can also predict the pressure ratio at a given mass flow rate and rotational speed conditions, and it can thus simulate the surging phenomenon $[3,16,28,29]$.

\subsection{Compressor Isentropic Efficiency Models}

\subsubsection{The Karlson Model}

Karlson [6] put forward an isentropic efficiency model for a large-scale marine compressor based on a fifth-order polynomial function of $\dot{m}$ and $\Pi$ with a total number of 21 model parameters. It should 
be noted that the theoretical foundation of the Karlson isentropic efficiency model is based on multiple regression analysis; therefore, its extrapolation accuracy cannot be guaranteed to some extent.

\subsubsection{The Guan Cong Model}

In addition to the mass flow rate model for the marine compressor, Guan et al. [34] also proposed a zonal isentropic efficiency model, the theoretical foundation of which is the approximately linear relationship existing between the dimensionless torque coefficient $\Gamma$ and flow coefficient $\phi$. The definition $\Gamma$ is as follows

$$
\Gamma=\frac{\frac{\dot{m} c_{\mathrm{p}} T_{\mathrm{in}}}{\omega \eta}\left(\Pi^{\frac{k-1}{k}}-1\right)}{\rho \pi r^{3} U^{2}}
$$

where $\omega(\omega=2 \pi N / 60)$ is the compressor angular velocity.

As similar to the mass flow rate model proposed by Guan et al. [34], the Guan Cong isentropic efficiency model also adopts the zonal modeling approach. As shown in Equation (25), the model parameters $p_{i}$ for the operating points within the current zone can be estimated through linear interpolation

$$
p_{i}=p_{i, \text { lower }}+\frac{\left(p_{i, \text { upper }}-p_{i, \text { lower }}\right)}{\left(N_{\text {upper }}-N_{\text {lower }}\right)}\left(N-N_{\text {lower }}\right)
$$

where $N_{\text {upper }}$ and $p_{i \text {,upper }}$ are the rotational speed, and corresponding model parameters for the upper boundary of the current zone; $p_{i, \text { lower }}$ and $N_{\text {lower }}$ are for the lower boundary.

To further improve the prediction accuracy, a correction procedure is carried out by the Guan Cong model based on the fact that the highest measured isentropic efficiency $\eta_{\max }$ of each iso-speed line can be approximated with a second-order function of rotational speed

$$
\eta_{\text {corr }}=\eta_{\text {non-corr }} \frac{\eta_{\max }(N)}{\eta_{\text {non-corr,max }}(N)}
$$

where $\eta_{\text {non-corr }}$ is the predicted isentropic efficiency without correction; $\eta_{\text {non-corr,max }}$ is the maximum value of predicted uncorrected isentropic efficiency at a rotational speed of $N ; \eta_{\text {corr }}$ is the corrected isentropic efficiency.

\subsubsection{The Zeng Tao Model}

Zeng et al. [36] proposed a mathematical model for predicting the compressor absorbed power $\dot{W}$ with the feature of low complexity. The theoretical basis of the Zeng Tao model is based on the Euler equation for turbomachinery. Meanwhile, various types of power losses caused by such as slip, windage and friction are also taken into account. In the Zeng Tao model, the two performance parameters power coefficient $C_{\text {power }}\left(C_{\text {power }}=\dot{W} / \dot{m}^{3}\right)$ and speed coefficient $C_{\text {speed }}\left(C_{\text {speed }}=\omega / \dot{m}\right)$, are defined. It was revealed by Zeng et al. [34] that a strong second-order relationship exists between $C_{\text {power }}$ and $C_{\text {speed }}$, and a strong linear relationship between the logarithm of $C_{\text {power }}$ and $C_{\text {speed }}$. Consequently, the isentropic efficiency can be estimated at given conditions of mass flow rate, angular velocity and pressure ratio according to its definition.

\subsubsection{The Hadef Model}

Based on the compressor isentropic efficiency model proposed by Martin and Talon [20], Hadef et al. [19] made appropriate improvements and assumptions and proposed the Hadef model. The theoretical basis of the Hadef model is the application of the Euler equation in the velocity triangle at the inlet and outlet of the compressor blade

$$
\Delta h_{\text {act }}=U^{2}-\frac{U \dot{m}}{\rho_{\text {out }} A_{\text {out }}} \cot \left(\beta_{\text {out }}\right)
$$


where $\Delta h_{\text {act }}$ is the actual specific enthalpy change across the compressor blade; $\rho_{\text {out }}, \beta_{\text {out }}$, and $A_{\text {out }}$ are the air density, outlet angle, and flow area at the outlet of compressor blade, respectively.

Based on the assumptions made by Hadef et al. [19], $\Delta h_{\text {act }}$ can be regarded as a linear function of mass flow rate at given rotational speed conditions, and thus Equation (27) can be rewritten as:

$$
\Delta h_{\mathrm{act}}=\left(b_{1} N+b_{2} N^{2}\right)-\left(a_{1} N+a_{2} N^{2}\right) \cdot \dot{m}
$$

After obtaining $\Delta h_{\text {act }}$ at given conditions of rotational speed and mass flow rate and the specific isentropic enthalpy change $\Delta h_{\text {is }}$ at given pressure ratio condition, the isentropic efficiency can be calculated with its definition: $\eta=\Delta h_{\text {is }} / \Delta h_{\text {act }}$.

\subsubsection{Llamas Model}

Llamas and Eriksson [28] further improved the Hadef model and proposed the Llamas model. Compared with the Hadef model, the Llamas model takes into account the change of air density at the inlet and outlet of the compressor blade as well as the influence of friction loss on $\Delta h_{\text {act }}$. The relationship between $\Delta h_{\text {act }}$ and $\dot{m}$ and $N_{\mathrm{n}}$ is expressed as follows

$$
\begin{gathered}
\Delta h_{\mathrm{act}}=\left(1+\frac{c}{\phi}\right)\left(b\left(N_{\mathrm{n}}\right)-a\left(N_{\mathrm{n}}\right) \dot{m}\right) \\
a\left(N_{\mathrm{n}}\right)=\frac{\Delta h_{\mathrm{act}, \max }}{\dot{m}_{\max }} \cdot \frac{a_{1} N_{\mathrm{n}}}{\left[1+a_{2} N_{\mathrm{n}}^{2}\right]^{3}} \dot{m}_{\max } \\
b\left(N_{\mathrm{n}}\right)=\Delta h_{\mathrm{act}, \max }\left(b_{1} N_{\mathrm{n}}^{2}+b_{2} N_{\mathrm{n}}^{3}\right) \\
N_{\mathrm{n}}=N / N_{\max }
\end{gathered}
$$

where $c$ is friction loss correction coefficient; $\Delta h_{\text {act,max }}, \dot{m}_{\max }$, and $N_{\max }$ is the maximum value of the measured $\Delta h_{\text {act }}, \dot{m}$, and $N$, respectively, which is used to normalize the base functions $a\left(N_{\mathrm{n}}\right), b\left(N_{\mathrm{n}}\right)$, and $N$. The derivation process of the base functions $a\left(N_{\mathrm{n}}\right)$ and $b\left(N_{\mathrm{n}}\right)$ is too long, which will not be introduced in this paper, and interested readers can refer to the original paper [28].

\section{Test Compressors, Comparative Methodology, and Error Evaluation Criteria}

To ensure unambiguous conclusions, the performance of various models was compared and analyzed for two marine compressors with different impeller outlet diameter, rotational, and flow ranges. The model numbers were A270-L59 and TCA88-25070, respectively, with their main technical parameters as shown in Table 1 . It should be noted that a larger number of measured sample data exists in the performance map of the A270-L59 compressor relative to that of the TCA88-25070 compressor, which was 289 for the formal and 54 for the later, and in addition, the distribution of iso-speed lines in the former was more intensive with an interval of 600 RPM. Consequently, the parameterization and the predictive result were expected to be better when the A270-L59 compressor was selected as the research object and the relevant comparative and analysis result was thus believed to be more representative. Nevertheless, the results obtained based on the TCA88-25070 compressor can be used to validate the comparative and analysis result obtained based on the A270-L59 compressor, to some extent.

Table 1. Main technical parameters of the A270-L59 and the TCA88-25070 marine compressors.

\begin{tabular}{ccc}
\hline Type & A270-L59 & TCA88-25070 \\
\hline Impeller Diameter $(\mathrm{m})$ & 0.59 & 0.893 \\
\hline Maximum Flow in the Map $\left(\mathrm{m}^{3} / \mathrm{s}\right)$ & 23 & 60 \\
\hline Maximum Speed in the Map $(\mathrm{RPM})$ & 16,800 & 11,763 \\
\hline
\end{tabular}


As mentioned in Section 2, the whole operating area of a compressor is divided into design, LS, HS, and LP area, so the predictive and extrapolation ability of various models was investigated, compared, and analyzed in these four areas separately. For the design operating area, all the measured sample data available in the performance map were used to parameterize the models and then each iso-speed line was predicted by the models to compare with the measured ones. For the LS or HS area, the measured sample data at the lowest or highest iso-speed line in the performance map were removed when parameterizing the models, and then the removed iso-speed line was extrapolated by the models for comparison with the measured one. It should be noted that the choking point on each iso-speed line was only measured and provided in the performance map of the TCA88-25070 compressor, so the analysis on the extrapolation ability of each model in the LP area was only performed on this compressor. For a certain mass flow rate model, its LP area extrapolation ability lies in whether the choking flow can be predicted accurately; on the other hand, the LP area extrapolation ability of an isentropic efficiency can be evaluated by investigating whether efficiency will drop to zero when the pressure ratio across the compressor decreases to one. In addition, it should be noted that the measured mass flow rate rather than predicted one by mass flow rate model was used when comparing the predictive ability of each isentropic efficiency model.

In this paper, the Curve Fitting toolbox in Matlab was adopted to determine the model parameters with the least square method, and the values given in the original papers were referred to when determining the initial guesses on these model parameters. In order to quantify the predictive ability of various compressor mass flow rate and isentropic efficiency model with their model parameters determined with the same measurement data set, the following four criteria for error evaluation were adopted in this paper, including corrected coefficient of determination $R_{\mathrm{c}}^{2}$, mean absolute percentage error $M A P E, P E B_{ \pm 5} \%$, and $P E B_{ \pm 10 \%}$, that is percentage of total data points within the error bounds \pm $5 \%$ and $\pm 10 \%$, respectively, with their definitions as follows $[12,13]$

$$
\begin{gathered}
R^{2}=1-\frac{\sum_{i=1}^{n}\left(y_{\text {meas }, i}-y_{\text {pred }, i}\right)^{2}}{\sum_{i=1}^{n}\left(y_{\text {meas }, i}-y_{\text {mean }}\right)^{2}} \\
R_{\mathrm{c}}^{2}=1-\frac{\left(1-R^{2}\right)(n-1)}{n-m-1} \\
R D_{i}=\frac{y_{\text {pred }, i}-y_{\text {meas }, i}}{y_{\text {meas }, i}} \\
M A P E=\frac{1}{n} \sum_{i=1}^{n}\left|R D_{i}\right| \\
P E B_{ \pm k \%}=\frac{m}{n}, m=\sum_{i=1}^{m} N_{k}(i), \text { where }\left|R D_{N_{k}}\right| \leq k \%
\end{gathered}
$$

where $R D_{i}$ is the relative deviation of $i t h$ measured data point; $y_{\text {meas }}$ is the measured value; $y_{\text {pred }}$ is the predicted value; $y_{\text {mean }}$ is the mean measured value; $n$ is the number of measured data points; and $m$ is the number of predictors ( $m=4$ in the Hadef model, for instance). 
The reason why the corrected coefficient of determination $R_{\mathrm{c}}^{2}$ was chosen rather than the coefficient of determination $R^{2}$ as the criteria for error evaluation is that $R^{2}$ is influenced by the number of measured sample data and predictors [12,13].

\section{Comparison and Analysis of Compressor Mass Flow Rate Model}

\subsection{Design Operating Area}

Figures 4 and 5 presents the comparison between the measurement sample data and the predicted iso-speed lines for the two compressors, respectively. Tables 2 and 3 give the corresponding error evaluation results for each model.

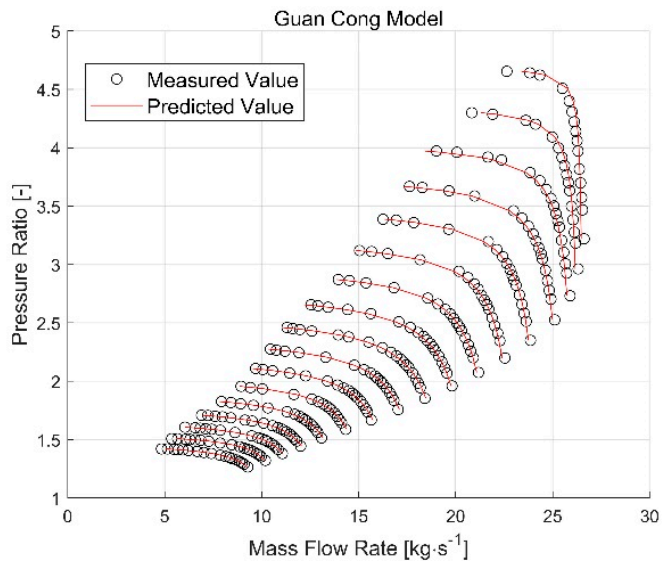

(a)

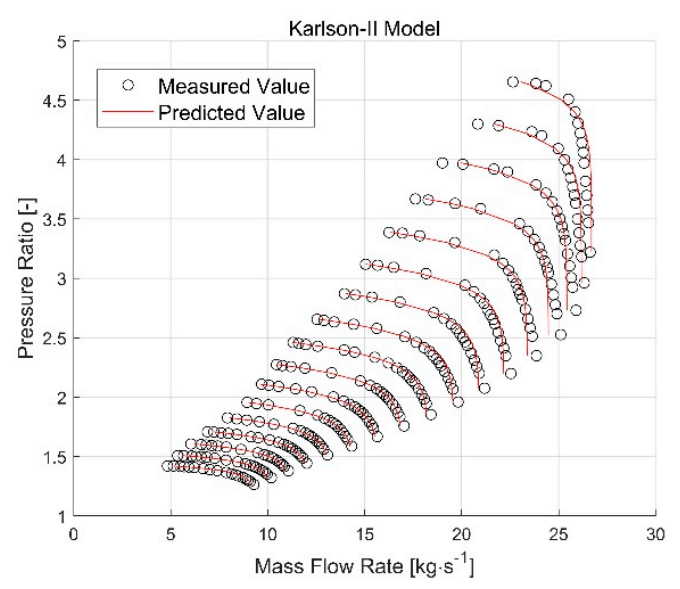

(c)

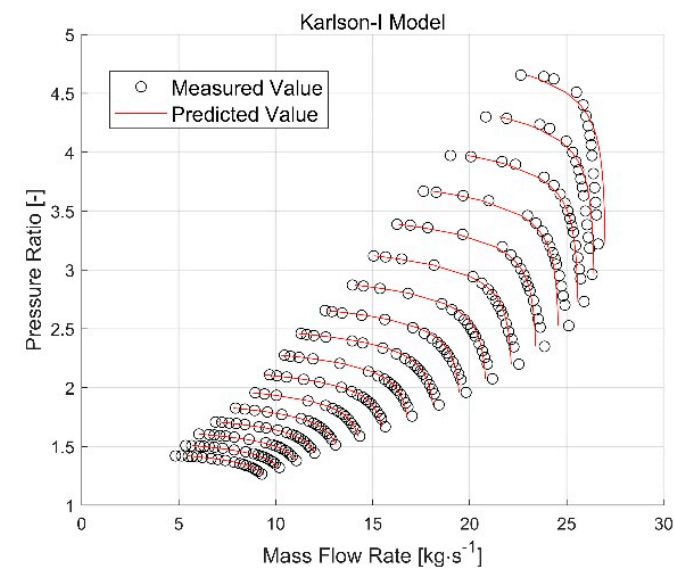

(b)

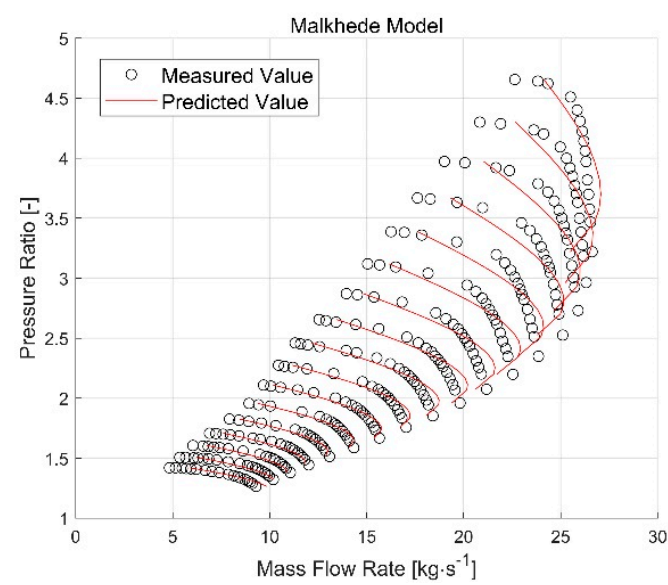

(d)

Figure 4. Cont. 


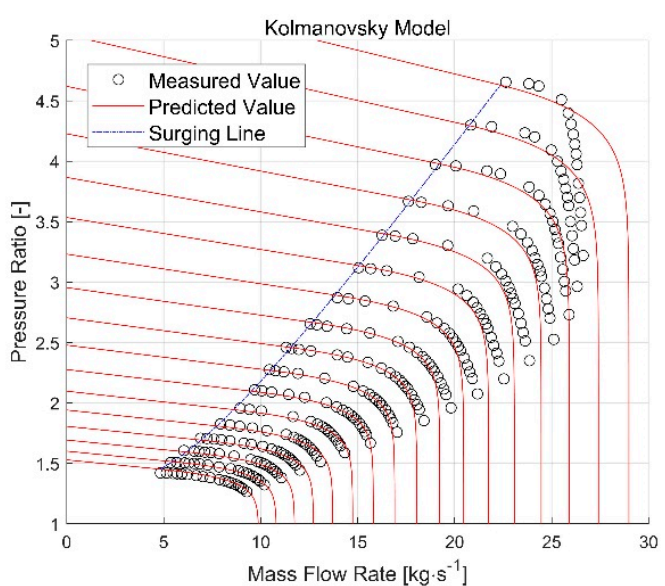

(e)

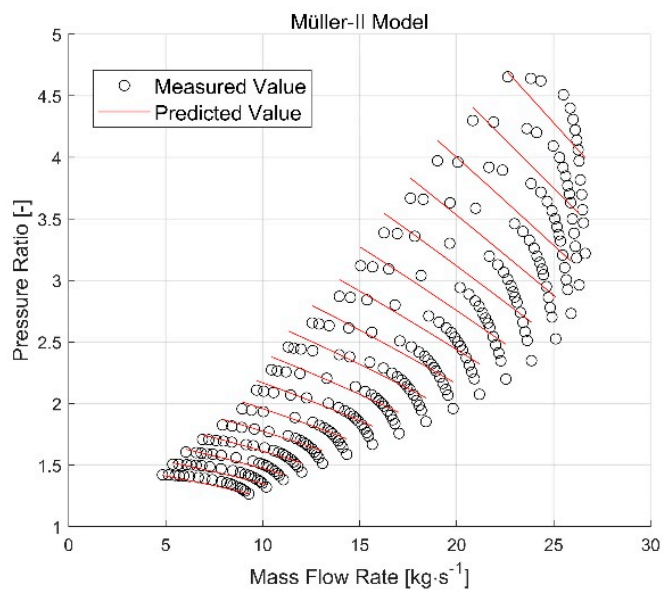

(g)

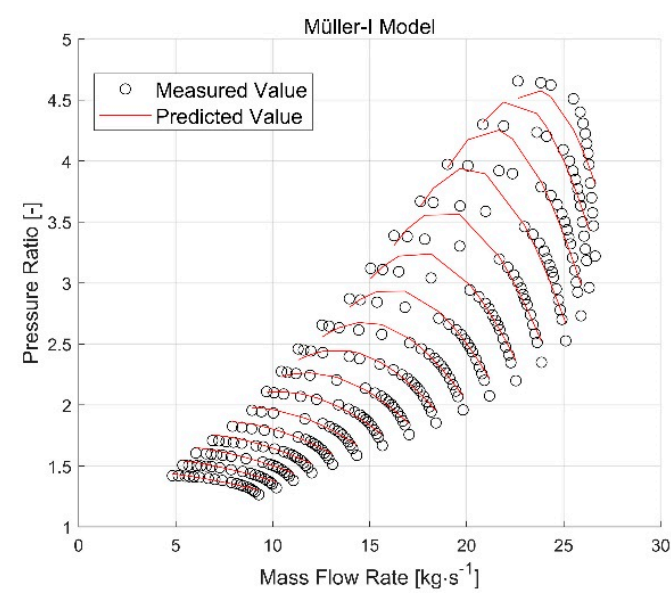

(f)

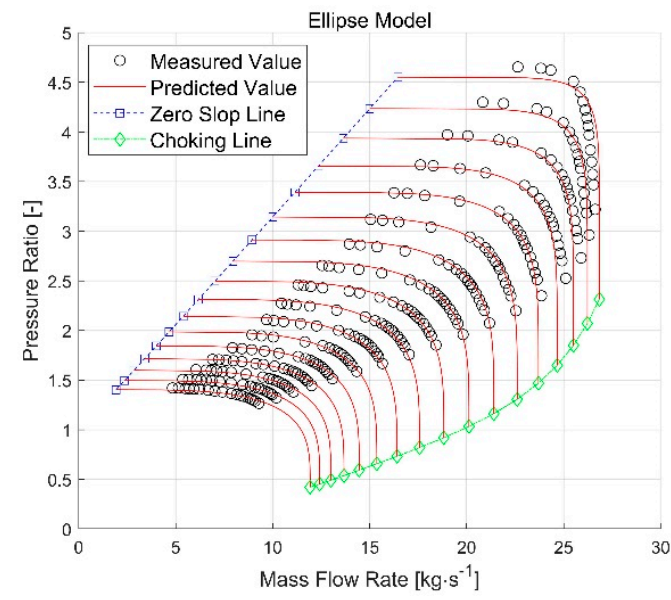

(h)

Figure 4. Prediction results of each compressor mass flow rate model in design operating area for the A270-L59 compressor: (a) the Guan Cong model; (b) the Karlson-I model; (c) the Karlson-II model; (d) the Malkhede model; (e) the Kolmanovsky model; (f) the Müller-I model; (g) the Müller-II model; and (h) the Leufvén and Llamas ellipse model.

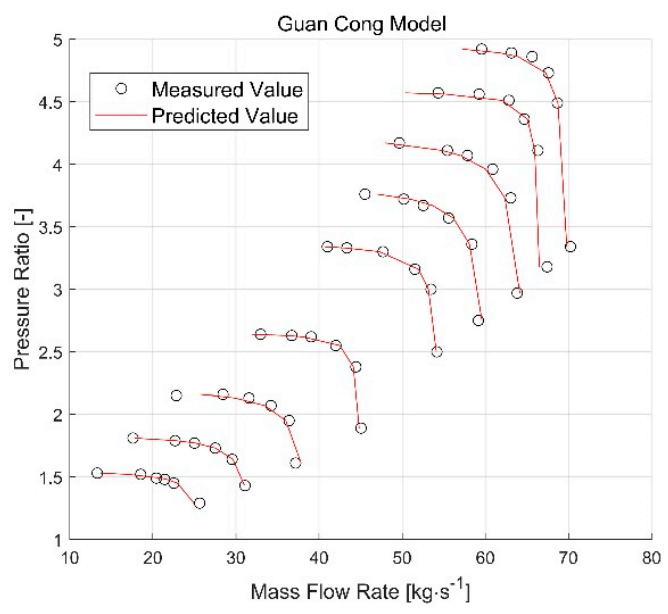

(a)

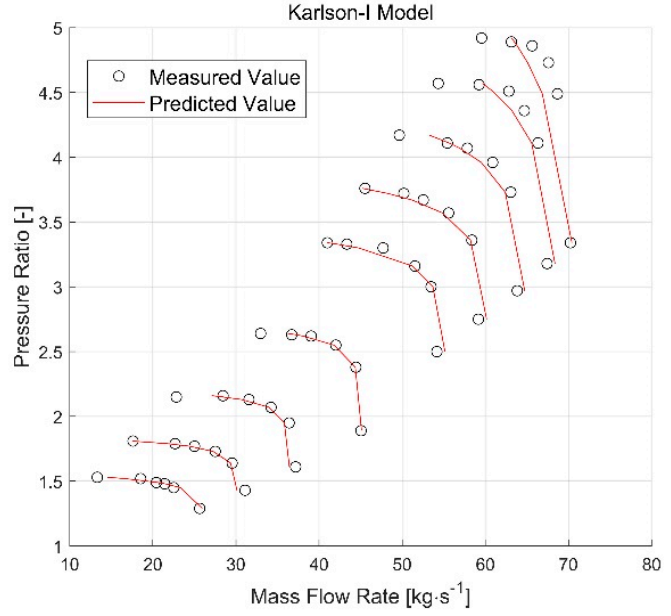

(b)

Figure 5. Cont. 


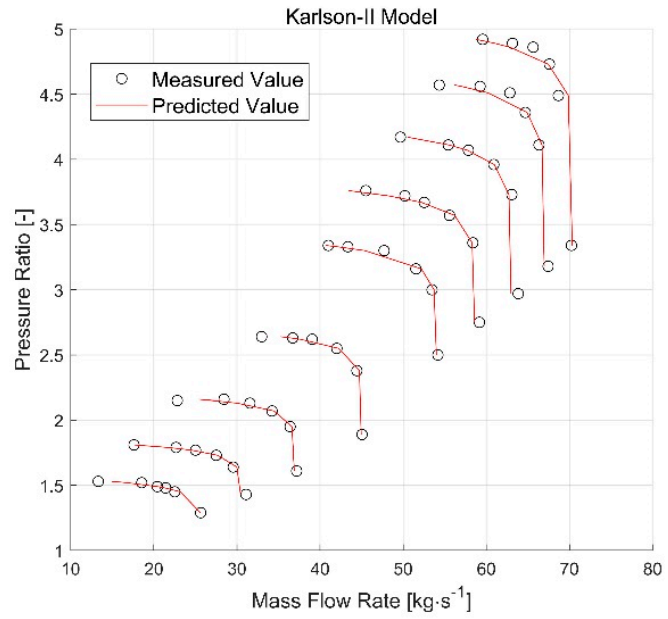

(c)

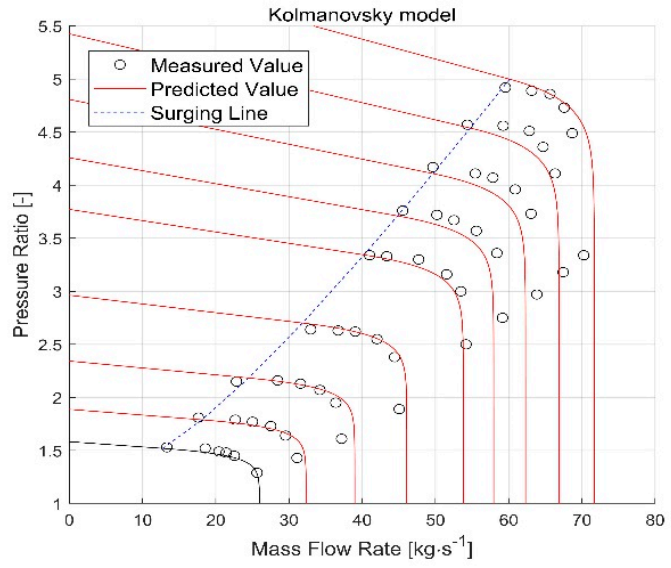

(e)

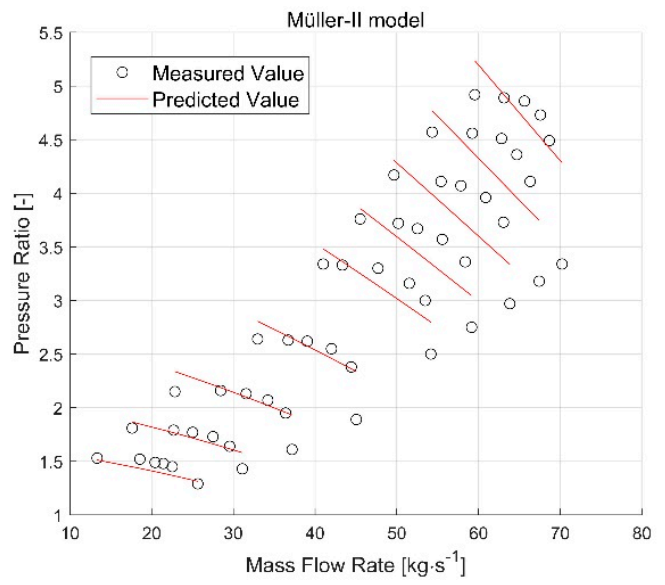

(g)

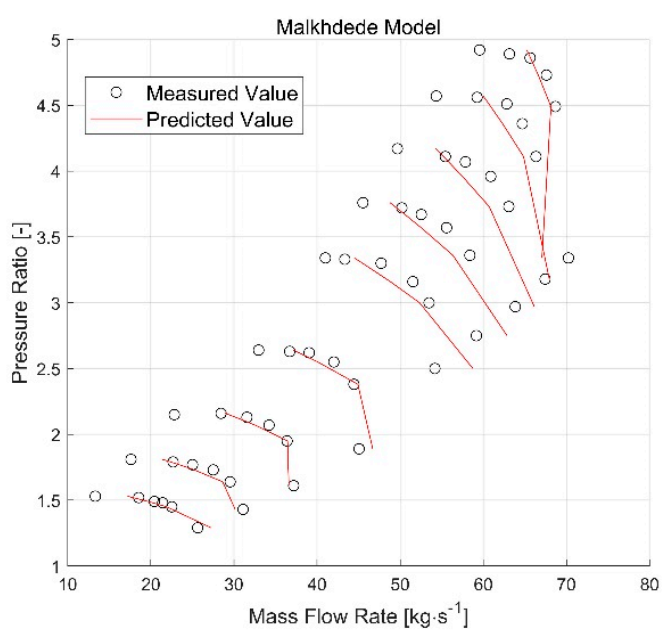

(d)

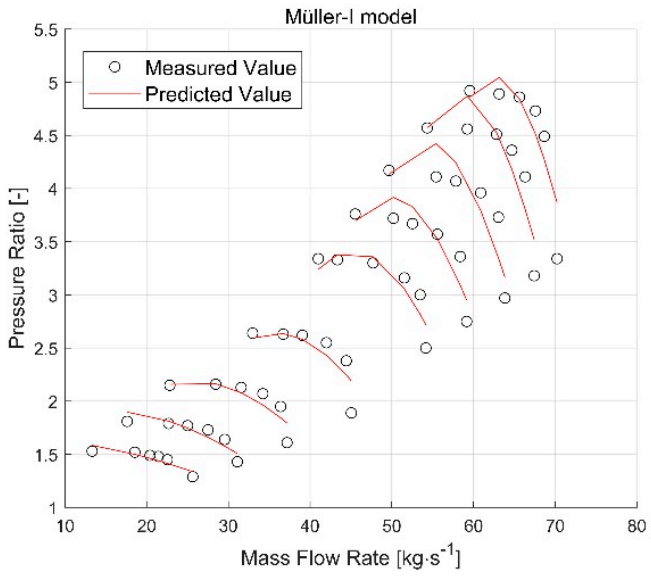

(f)

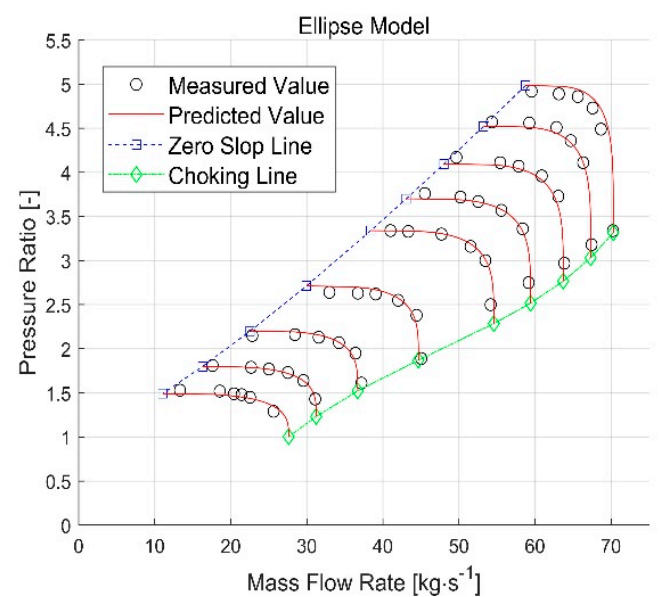

(h)

Figure 5. Prediction results of each compressor mass flow rate model in design operating area for the TCA88-25070 compressor: (a) the Guan Cong model; (b) the Karlson-I model; (c) the Karlson-II model; (d) the Malkhede model; (e) the Kolmanovsky model; (f) the Müller-I model; (g) the Müller-II model; and (h) the Leufvén and Llamas ellipse model. 
Table 2. Error evaluation results of each compressor mass flow rate model in the design operating area for the A270-L59 compressor.

\begin{tabular}{ccccc}
\hline Mathematical Model & $\boldsymbol{R}_{\mathbf{c}}^{\mathbf{2}(-)}$ & MAPE (\%) & $\boldsymbol{P E B}_{ \pm \mathbf{5} \% \mathbf{( \% )}}$ & $\boldsymbol{P E B}_{ \pm \mathbf{1 0} \% \mathbf{( \% )}}$ \\
\hline Guan Cong model & 0.9996 & 0.5565 & 100 & 100 \\
Karlson-I model & 0.9989 & 1.0055 & 98.9619 & 99.6540 \\
Karlson-II model & 0.9989 & 0.9106 & 99.3080 & 100 \\
Malkhede model & 0.9905 & 3.2034 & 82.0069 & 97.9239 \\
Kolmanovsky model & 0.9890 & 3.3850 & 82.0069 & 92.7336 \\
Müller-I model & 0.9864 & 2.1315 & 89.2734 & 98.9619 \\
Müller-II model & 0.9714 & 3.5198 & 77.5087 & 95.8478 \\
Ellipse model & 0.9133 & 6.8087 & 77.8547 & 84.7751 \\
\hline
\end{tabular}

Table 3. Error evaluation results of each compressor mass flow rate model in the design operating area for the TCA88-25070 compressor.

\begin{tabular}{ccccc}
\hline Mathematical Model & $\boldsymbol{R}_{\mathbf{c}}^{\mathbf{2}(-)}$ & $\boldsymbol{M A P E}(\mathbf{\%})$ & $\mathbf{P E B}_{ \pm \mathbf{5} \%} \mathbf{( \% )}$ & $\boldsymbol{P E B}_{ \pm \mathbf{1 0} \%} \mathbf{( \% )}$ \\
\hline Guan Cong model & 0.9926 & 2.2779 & 88.8889 & 98.1481 \\
Karlson-I model & 0.9870 & 2.9648 & 85.1852 & 96.2963 \\
Karlson-II model & 0.9916 & 2.5279 & 90.7407 & 94.4444 \\
Malkhede model & 0.9703 & 5.3878 & 66.6667 & 90.7407 \\
Kolmanovsky model & 0.9618 & 6.7642 & 57.4074 & 75.9259 \\
Müller-I model & 0.9722 & 4.3696 & 66.6667 & 90.7407 \\
Müller-II model & 0.9456 & 6.2761 & 51.8519 & 83.3333 \\
Ellipse model & 0.9191 & 7.6050 & 64.8148 & 70.3704 \\
\hline
\end{tabular}

By observing the comparative and error evaluation results shown in Figures 4 and 5 and Tables 2 and 3, it can be found that in general, all the investigated models were capable of characterizing the changing trend between mass flow rate and pressure ratio for both compressors in the design operating area, but with different predictive accuracy. However, by comparing Tables 2 and 3 , it can be seen that the predictive accuracy of each mass flow rate model dropped moderately when the research object changed from the A270-L59 to the TCA88-25070 marine compressor. This is perhaps due to the fact that a relatively smaller number of measured sample data exists in the performance map of the TCA88-25070 compressor relative to the A270-L59 compressor, which is 54 for the former, and 289 for the later, as mentioned in Section 4. Consequently, the overall predictive accuracy of the compressor models could be polluted to some extent in the existence of several predictive points with relatively large errors, like for the TCA88-25070 compressor. Nevertheless, in general, the ranking of these models in terms of predictive accuracy was found to be consistent for the two different marine compressors by analyzing the obtained error evaluation results, which demonstrates the validity of the results given in this paper, to some extent.

Among these models, the Guan Cong, Karlson-I, and Karlson-II models were the top three mass flow rate models with MAPEs around 1\% for the A270-L59 compressor and around $2.5 \%$ for the TCA88-25070 marine compressor; additionally, they are also capable of characterizing the changing trend of the iso-speed line when approaching the choking point. Of the top three models, the Guan Cong model achieved the best predictive accuracy, mainly owing to its zonal modeling methodology.

Although it was revealed by Leufvén and Eriksson [3] that desirable predictive accuracy can be achieved with the Leufvén and Llamas ellipse model, the error evaluation results shown in Tables 2 and 3 indicate that the predictive accuracy of this model is undesirable for the two marine compressors investigated in this paper. This phenomenon is caused mainly due to its theoretical foundation: A super-ellipse is utilized to approximate the iso-speed line, and the maximum pressure ratio in the design operating area should not exceed the pressure ratio at ZSL, i.e., $\Pi_{\mathrm{Zs}}$, otherwise, the straight line as shown in Equation (23) will be used to calculate the mass flow rate. Nevertheless, it cannot be guaranteed that the pressure ratio of all the measured sample data would be less than $\Pi_{\mathrm{zs}}$ at a given 
rotational speed condition. As a result, for those measured sample data with a pressure ratio larger than $\Pi_{z \mathrm{z}}$ the predicted mass flow rates will obviously deviate from the measured ones, which impairs the model predictive accuracy significantly. If those measured sample data are removed, the predictive accuracy of the Leufvén and Llamas ellipse model will improve greatly. Based on the above analysis, it can be concluded that the Leufvén and Llamas ellipse model has a relatively high requirement for the quality of measured sample data, thus weakening its practicability in engineering practices.

Despite the relatively lower predictive accuracy in the whole design operating area, it was found that for both the Müller-I and -II models, satisfactory predictive accuracy could be achieved under low rotational speed conditions, and the reason for this phenomenon is mainly owed to their theoretical foundation as introduced in Sections 3.1.5 and 3.1.6. For the Malkhede model, its $P E B_{ \pm 10 \%}$ was larger than $90 \%$, and $M A P E$ less than $5.5 \%$ for both compressors, so its predictive ability was considered to be satisfactory if the requirement on predictive accuracy is not extremely high. However, it can be observed in Figures 4 and 5, that after crossing a certain operating point, the mass flow rate will decrease with a decrease in pressure ratio, which is unrealistic and violates the actual changing trend. Although the Kolmanovsky model is capable of characterizing the changing trend of the iso-speed line when approaching the choking point, its predictive accuracy was relatively low among the investigated models, especially for the TCA88-25070 compressor.

\subsection{Off-Design Operating Area}

\subsubsection{Low-Pressure Ratio (LR) Area}

Figure 6 presents the changing trend of choking pressure ratio $\Pi_{\mathrm{ch}}$ and choking volume flow rate $\dot{V}_{\text {ch }}$ with rotational speed for the TCA88-25070 marine compressor. As observed in Figure $6 a, \Pi_{\text {ch }}$ increased monotonously with the rotational speed and all the measured values of $\Pi_{\mathrm{ch}}$ were greater than one. With a further decrease in rotational speed $\Pi_{\mathrm{ch}}$ will decrease monotonously, and at a certain rotational speed, the condition of $\Pi_{\mathrm{ch}}$ will become less than one. The research carried out by Leufvén and Eriksson [29] revealed that $\Pi_{\mathrm{ch}}$ is approximately equal to 0.5 when the compressor rotational speed decreases to zero. Similarly, as observed in Figure $6 \mathrm{~b}, \dot{V}_{\mathrm{ch}}$ also increased monotonously with compressor rotational speed, but the increasing magnitude decreased gradually.

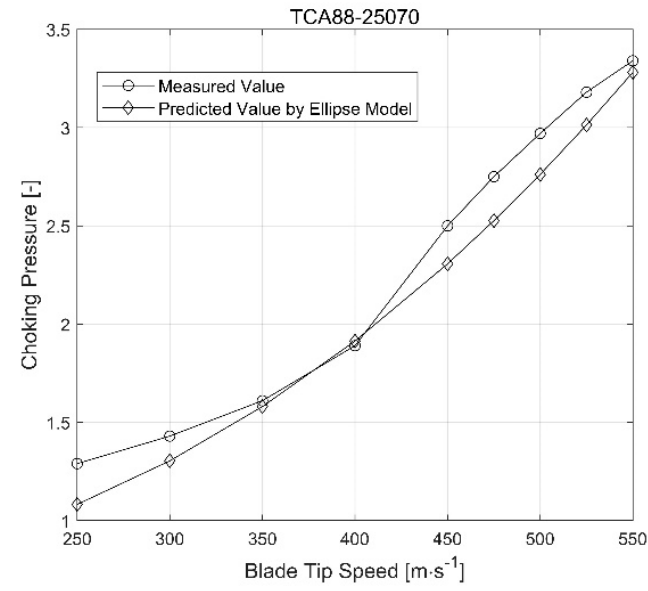

(a)

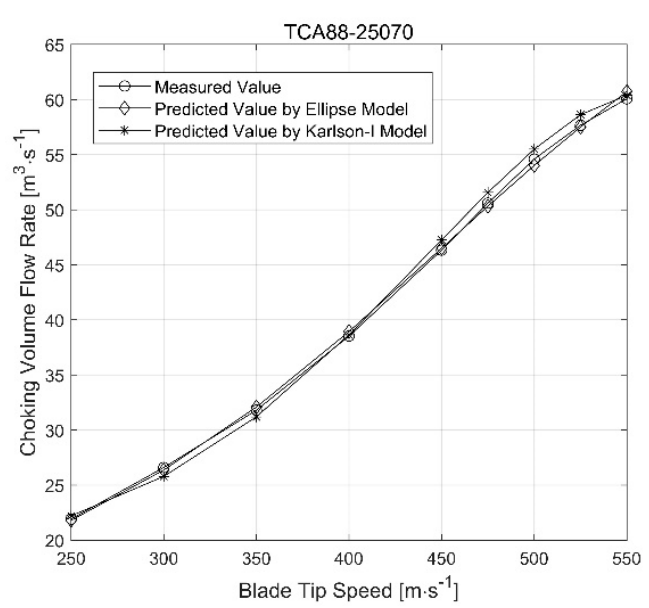

(b)

Figure 6. Measured and predicted choking pressure and volume flow rate: (a) choking pressure; (b) choking volume flow rate.

Although the predictive accuracy of the Leufvén and Llamas ellipse model is relatively undesirable in the design operating area for both compressors, special base functions are defined in this model to characterize the changing trend of choking pressure ratio and choking mass (or volume) flow rate with the rotational speed. As seen in Figures 4 and 5, the Leufvén and Llamas ellipse model was 
able to describe the changing trend of the iso-speed line when approaching the choking point, i.e., its slope gradually approached infinity. In addition to the Leufvén and Llamas ellipse model, the Karlson-I, Karlson-II, and Kolmanovsky models utilize the curve characteristic of the exponential function to characterize the compressor choking phenomenon. As can be seen in Figures 4 and 5, for the Kolmanovsky model, the predicted iso-speed line entered into the choking area either too early or late; on the other hand, the Karlson-I and Karlson-II models appeared to have similar LP area extrapolation capabilities. So, in this section, only the Leufvén and Llamas ellipse models and the Karlson-I model were compared and analyzed in terms of LR area extrapolation ability.

Besides the measured data points, the choking pressure ratio predicted by the Leufvén and Llamas ellipse model is also depicted in Figure 6a. In Figure $6 \mathrm{~b}$, the choking volume flow rate predicted by the Leufvén and Llamas ellipse model and the Karlson-I model is depicted along with the measured sample data. As can be observed in Figure 6, both of the two models were able to accurately characterize the changing trend of choking volume flow rate at rotational speeds; in addition, the Leufvén and Llamas ellipse model was also capable of describing the changing trend of choking pressure ratio with speed, which gave it unique superiority relative to the Karlson-I model.

Table 4 presents the measured choking volume flow rate $\dot{V}_{\text {ch,meas, }}$, the predicted values $\dot{V}_{\text {ch,ell }}$ by the Leufvén and Llamas ellipse model, and $\dot{V}_{\text {ch,exp }}$ by the Karlson-I model as well as the corresponding relative error $R D_{\text {ell }}$ and $R D_{\text {exp }}$, under each speed condition. As observed in Table $4, R D_{\text {ell }}$ is generally lower than $R D_{\text {exp }}$, and the MAPE of the Leufvén and Llamas ellipse model is also lower than that of the Karlson-I model. In addition, since the Leufvén and Llamas ellipse model was capable of predicting the choking pressure ratio accurately and reasonably, the validity of the changing trend of the iso-speed line was guaranteed to some extent when approaching the choking point. In conclusion, although the predictive accuracy of the Leufvén and Llamas ellipse model is undesirable in the design operating area, its LR area extrapolation ability was the best among the compressor mass flow rate models investigated in this paper.

Table 4. Measured and predicted choking volume flow rates and prediction errors at each rotational speed condition for the TCA88-25070 compressor.

\begin{tabular}{cccccc}
\hline$U(\mathrm{~m} / \mathbf{s})$ & $\dot{V}_{\text {ch,meas }}\left(\mathbf{m}^{3} / \mathbf{s}\right)$ & $\dot{V}_{\text {ch,ell }}\left(\mathbf{m}^{3} / \mathbf{s}\right)$ & $R D_{\text {ell }}(\%)$ & $\dot{V}_{\text {ch,exp }}\left(\mathbf{m}^{3} / \mathbf{s}\right)$ & $R D_{\exp }(\%)$ \\
\hline 550 & 60.07 & 60.72 & 1.0806 & 60.39 & 0.5290 \\
525 & 57.67 & 57.50 & -0.2945 & 58.63 & 1.6624 \\
500 & 54.60 & 54.02 & -1.0713 & 55.49 & 1.6391 \\
475 & 50.60 & 50.32 & -0.5494 & 51.56 & 1.9006 \\
450 & 46.33 & 46.51 & 0.3834 & 47.26 & 2.0006 \\
400 & 38.53 & 38.94 & 1.0654 & 38.65 & 0.3122 \\
350 & 31.80 & 32.09 & 0.9161 & 31.20 & -1.8805 \\
300 & 26.60 & 26.37 & -0.8529 & 25.79 & -3.0360 \\
250 & 21.93 & 21.81 & -0.5696 & 22.21 & 1.2951 \\
\hline MAPE (\%) & & 0.7537 & & 1.5839 \\
\hline
\end{tabular}

\subsubsection{Low Speed (LS) Area}

In Figures 7 and 8, the HS area extrapolation results of each model are compared with the A270-L59 and the TCA88-25070 compressor as modeling objects, respectively, and Tables 5 and 6 provide corresponding error evaluation results. 


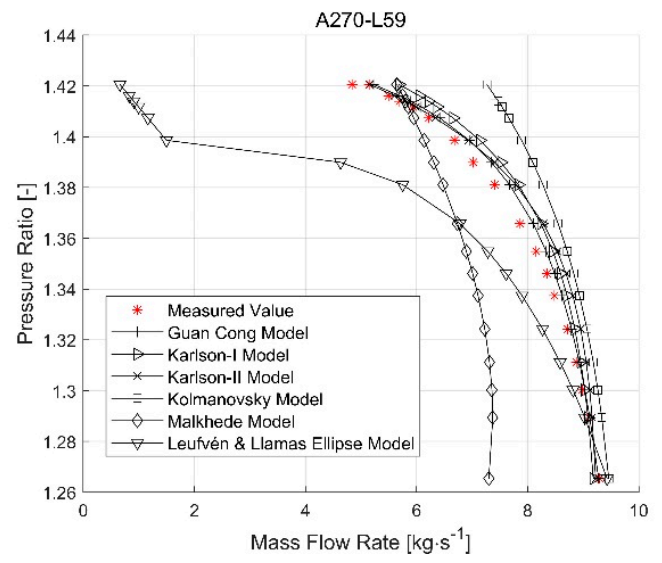

(a)

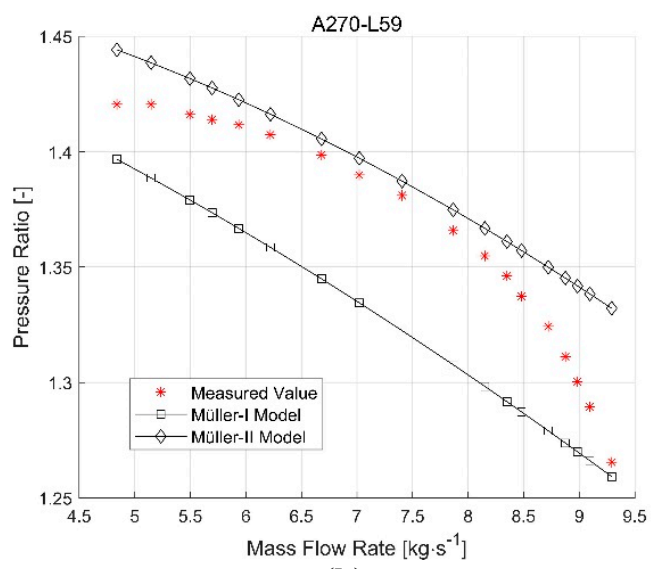

(b)

Figure 7. LS area extrapolation results for each compressor mass flow rate model for the A270-L59 compressor: (a) model structure of $\dot{m}=f(N, \Pi)$; (b) model structure of $\Pi=f(N, \dot{m})$.

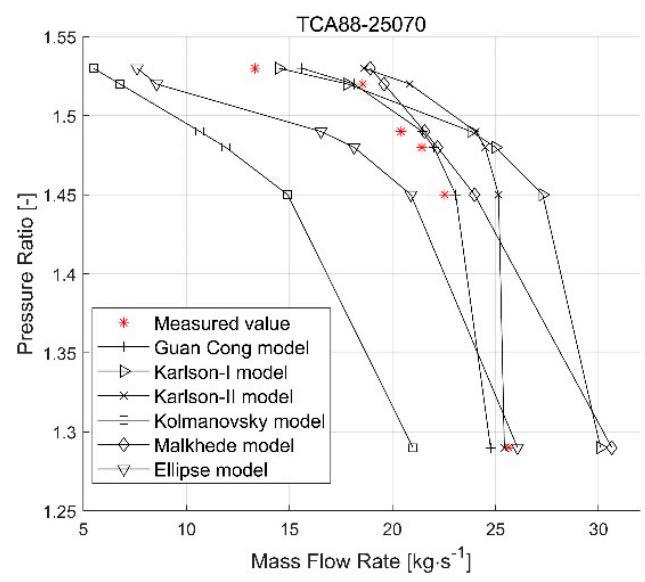

(a)

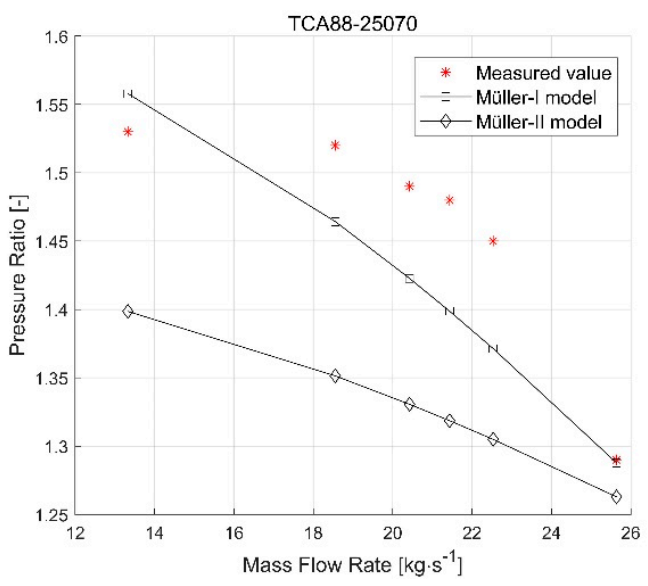

(b)

Figure 8. LS area extrapolation results for each compressor mass flow rate model for the TCA88-25070 compressor: (a) model structure of $\dot{m}=f(N, \Pi)$; (b) model structure of $\Pi=f(N, \dot{m})$.

Table 5. Error evaluation results of the LS area extrapolation results of each compressor mass flow rate model for the A270-L59 compressor.

\begin{tabular}{ccccc}
\hline & Guan Cong Model & Karlson-I Model & Karlson-II Model & Kolmanovsky Model \\
\hline MAPE $(\%)$ & 2.5277 & 5.5278 & 2.9697 & 16.3360 \\
MAPE $(\%)$ & Malkhede model & Ellipse model & Müller-I model & Müller-II model \\
\hline
\end{tabular}

Table 6. Error evaluation results of LS area extrapolation results of each compressor mass flow rate model for the TCA88-25070 compressor.

\begin{tabular}{ccccc}
\hline & Guan Cong Model & Karlson-I Model & Karlson-II Model & Kolmanovsky Model \\
\hline MAPE $(\%)$ & 5.4494 & 14.0635 & 16.0772 & 44.4080 \\
& Malkhede model & Ellipse model & Müller-I model & Müller-II model \\
MAPE $(\%)$ & 13.7704 & 23.3626 & 3.5147 & 8.8979 \\
\hline
\end{tabular}

It can be found from the comparative and error evaluation results that the LS area extrapolation ability of the Guan Cong, Karlson-I, Karlson-II, Müller-I, and Müller-II models is obviously better than the other models when the A270-L59 compressor was selected as modeling object. However, it dropped significantly for both the Karlson-I and Karlson-II models for the TCA88-25070 compressor, 
which is maybe due to the relatively smaller number of measured data points and the sparse iso-speed lines available in the performance map of this particular compressor.

Among these models, the Guan Cong model manifests a satisfactory LS area extrapolation accuracy for both compressors with a MAPE of less than $5.5 \%$, which is mainly attributed to its zonal modeling methodology as well as the fact that air is effectively incompressible under low-speed conditions, and thus, the changing trend between mass flow rate and pressure ratio will not change significantly. It is interesting to note that the Müller-I model achieved the best extrapolation ability for both compressors with a MAPE of $1.5994 \%$ for the A270-L59 and 3.5147\% for the TCA88-25070, which was mainly due to its theoretical foundation. However, it should be noted that this model belongs to the second type of mass flow rate model as shown in Equation (8), which is not suitable for the dynamic simulation modeling of turbocharged diesel engines. It can be observed from Figures 7 and 8 that several predicted values by the Leufvén and Llamas ellipse model significantly deviate from the measured ones because of the greater pressure ratio than that at ZSL. For the Kolmanovsky and Malkhede models, their LS area extrapolation accuracy was undesirable, which will be not discussed further.

\subsubsection{High Speed (HS) Area}

It can be observed from the comparative and error evaluation results, as shown in Figures 9 and 10 and Tables 7 and 8, that all the investigated models achieved satisfactory HS area extrapolation accuracy except for the Guan Cong and Ellipse models. It is interesting to note that relative to its LS area extrapolation accuracy, the Guan Cong model dropped significantly in the HS area with a MAPE as high as $28.7346 \%$ for the A270-L59 compressor and $17.5797 \%$ for the TCA88-25090 compressor; in addition, for the TCA88-25090 compressor, the predicted mass flow rate decreased with a decrease in pressure ratio, which is unrealistic and violates the actual changing trend. The reason causing the inferior HS area extrapolation accuracy for the Guan Cong model may be due to the fact that when extrapolating to the HS area, the model parameters corresponding to the highest iso-speed lines are still adopted, which cannot capture the influence of rotational speed change on the fluid physical properties, especially the compressibility. Similar to the LS area, several predicted values by the Leufvén and Llamas ellipse model significantly deviated from the measured values. In contrast to the LS area, satisfactory extrapolation accuracy was achieved by both the Karlson-I and Karlson-II models in the HS area, with a MAPE less than 2.6\% for the A270-L59 compressor and 5\% for TCA88-25070 compressor, which demonstrates their stable and robust HS area extrapolation capability to some extent.

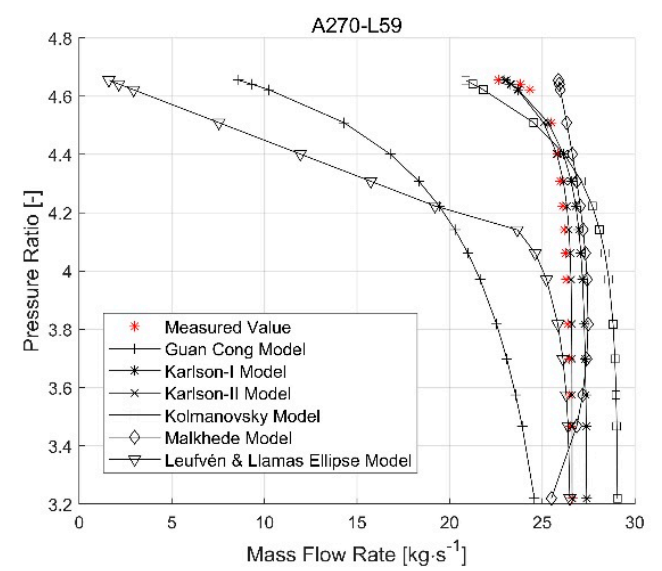

(a)

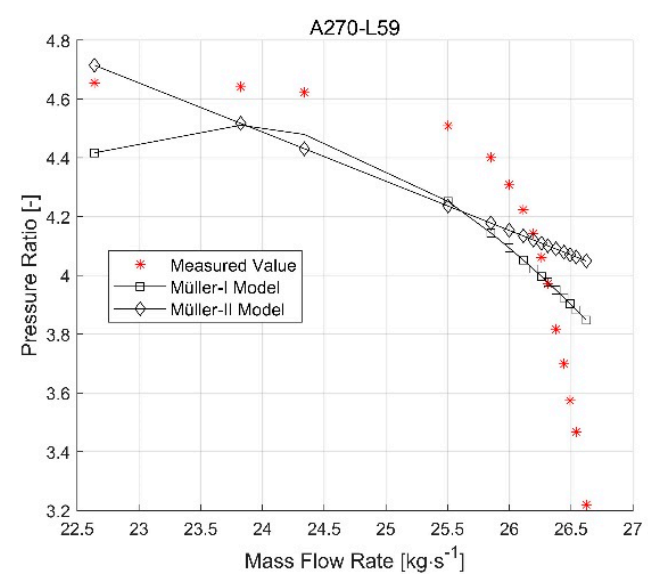

(b)

Figure 9. HS extrapolation results for each compressor mass flow rate model for the A270-L59 compressor: (a) model structure of $\dot{m}=f(N, \Pi)$; (b) model structure of $\Pi=f(N, \dot{m})$. 


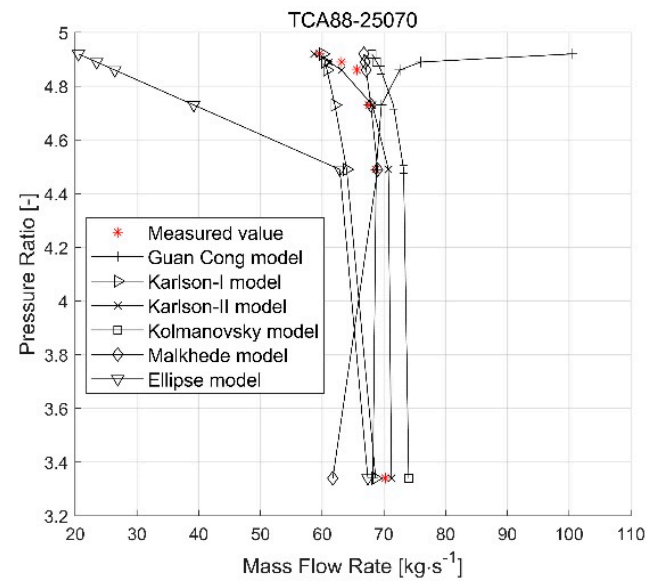

(a)

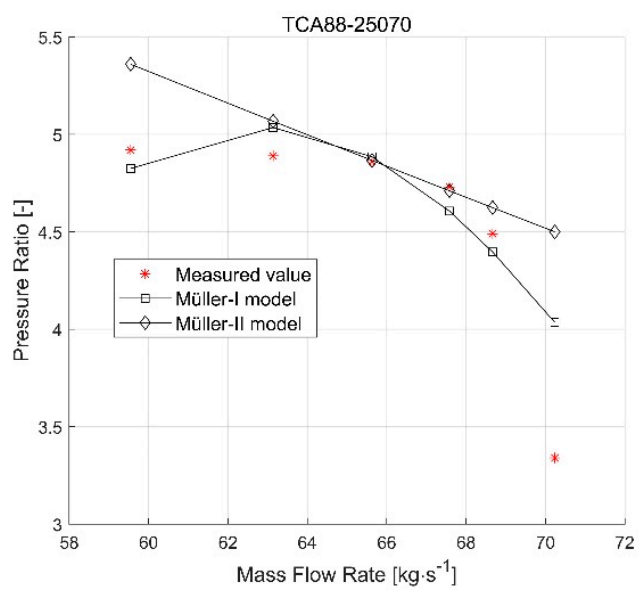

(b)

Figure 10. HS area extrapolation results for each compressor mass flow rate model for TCA88-25070 compressor: (a) model structure of $\dot{m}=f(N, \Pi)$; (b) model structure of $\Pi=f(N, \dot{m})$.

Table 7. Error evaluation results of HS area extrapolation results of each compressor mass flow rate model for the A270-L59 compressor.

\begin{tabular}{ccccc}
\hline & Guan Cong Model & Karlson-I Model & Karlson-II Model & Kolmanovsky Model \\
\hline MAPE $(\%)$ & 28.7346 & 2.5749 & 1.0053 & 7.6798 \\
MAPE $(\%)$ & Malkhede model & Ellipse model & Müller-I model & Müller-II model \\
\hline
\end{tabular}

Table 8. Error evaluation results of HS area extrapolation results of each compressor mass flow rate model for the TCA88-25070 compressor.

\begin{tabular}{ccccc}
\hline & Guan Cong Model & Karlson-I Model & Karlson-II Model & Kolmanovsky Model \\
\hline MAPE $(\%)$ & 17.5797 & 4.9733 & 2.2754 & 7.8512 \\
MAPE $(\%)$ & Malkhede model & Ellipse model & Müller-I model & Müller-II model \\
\hline
\end{tabular}

\section{Comparison and Analysis of Compressor Isentropic Efficiency Model}

\subsection{Design Operating Area}

Figures 11 and 12 present the prediction results for each compressor isentropic efficiency model along with the measured data points for the A270-L59 and TCA88-25070 compressors, respectively. Tables 9 and 10 provide the corresponding error evaluation results. In order to clearly display and compare the prediction results, only part of the iso-speed lines are depicted in Figures 11 and 12. 


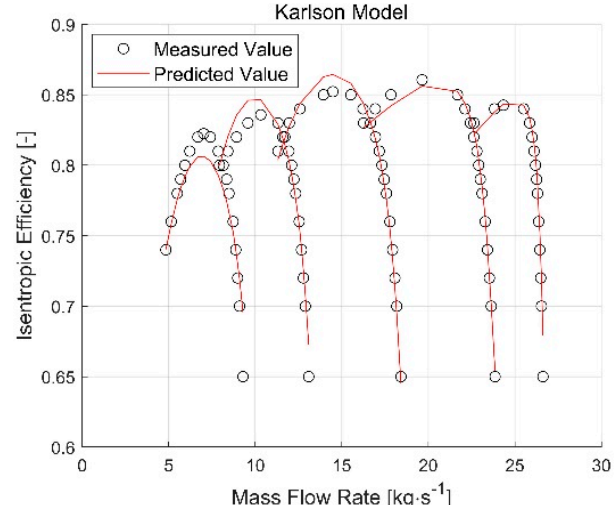

(a)

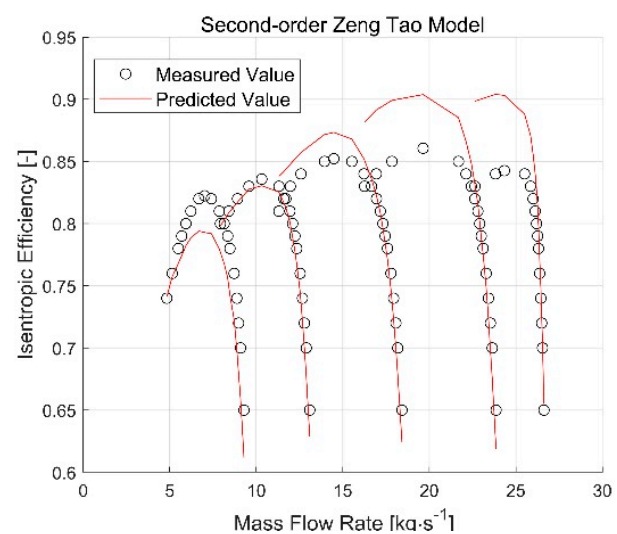

(c)

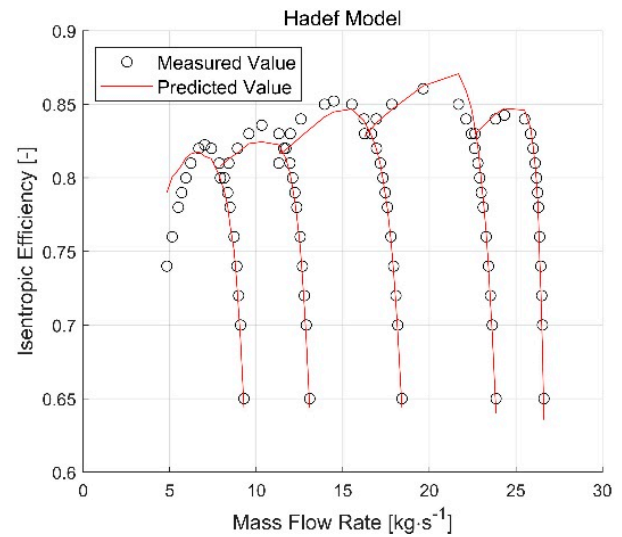

(e)

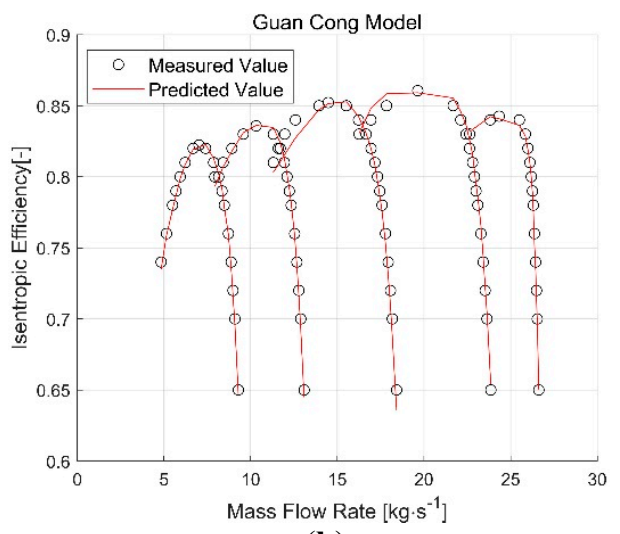

(b)

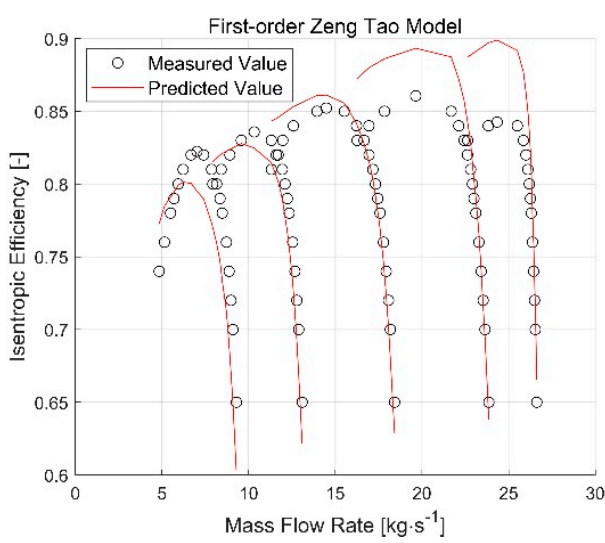

(d)

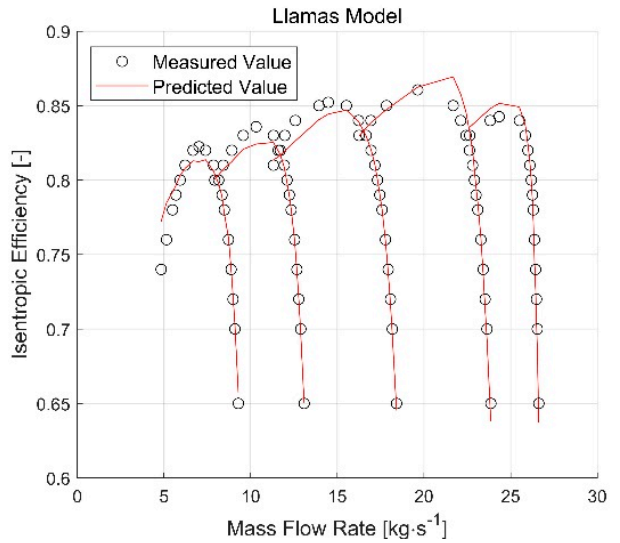

(f)

Figure 11. Prediction results of each compressor isentropic efficiency model in design operating area for the A270-L59 compressor: (a) the Karlson model; (b) the Guan Cong model; (c) the Second-order Zeng Tao model; (d) the First-order Zeng Tao model; (e) the Hadef model; (f) and the Llamas model. 


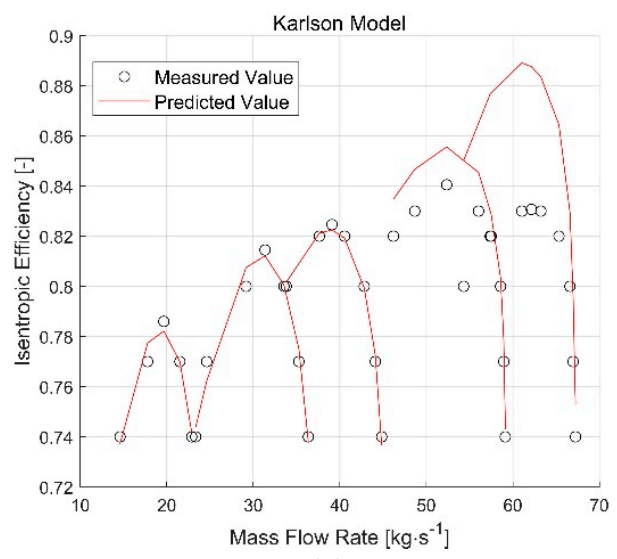

(a)

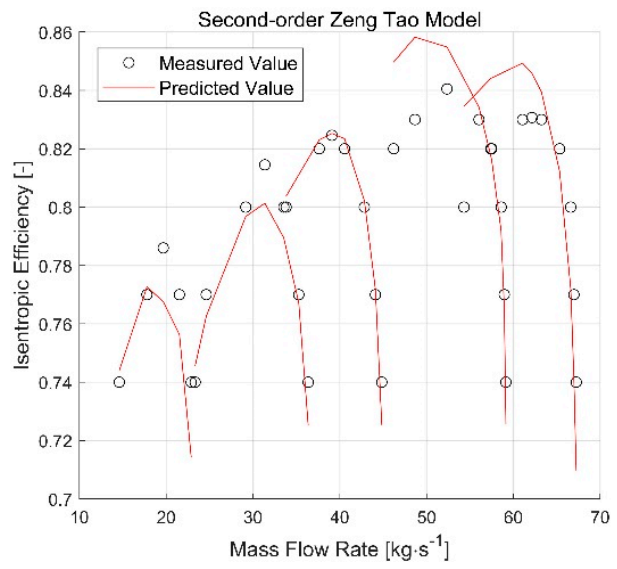

(c)

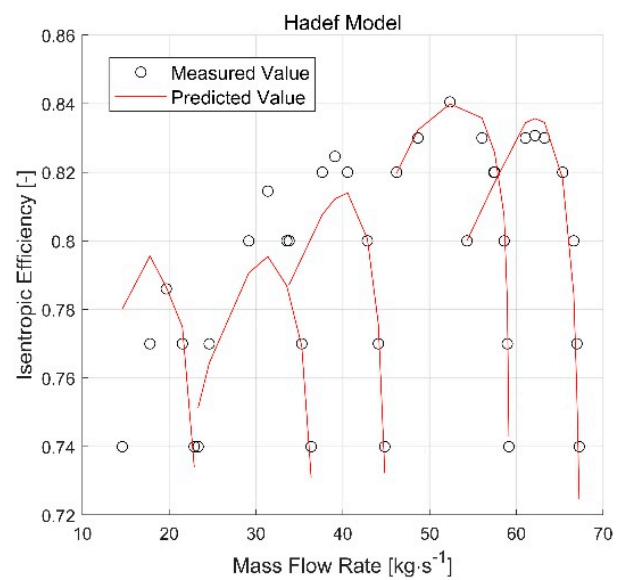

(e)

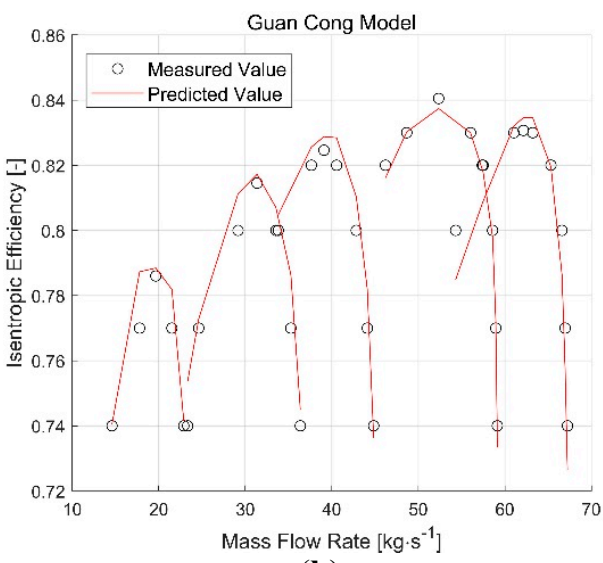

(b)

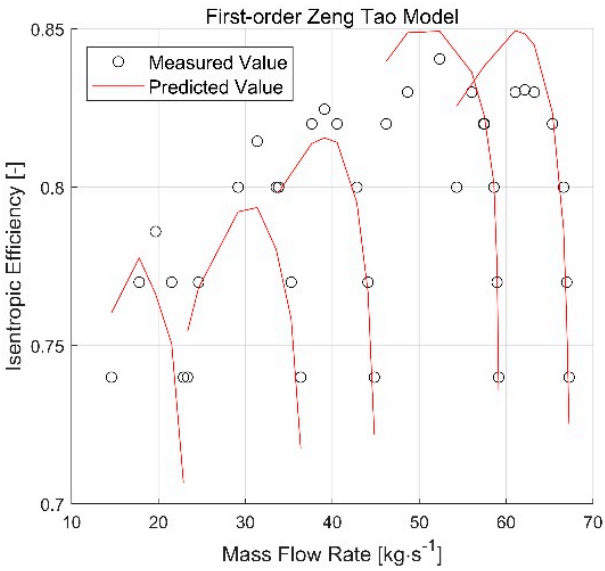

(d)

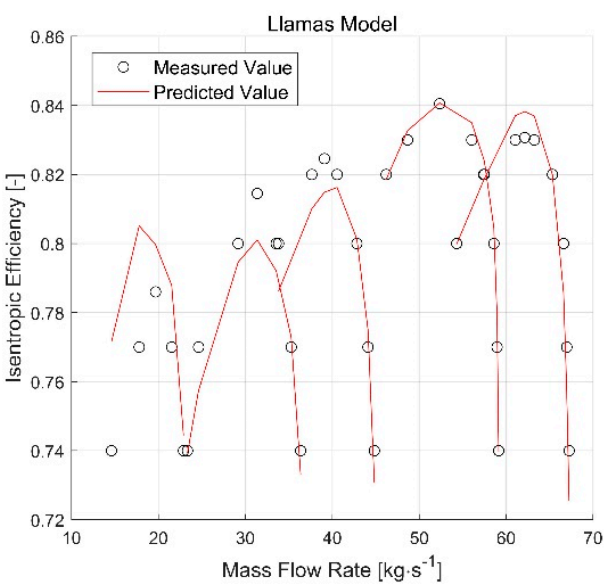

(f)

Figure 12. Prediction results of each compressor isentropic efficiency model in the design operating area for the TCA88-25070 compressor: (a) the Karlson model; (b) the Guan Cong model; (c) the Second-order Zeng Tao model; (d) the First-order Zeng Tao model; (e) the Hadef model; (f) and the Llamas model. 
Table 9. Error evaluation results of each compressor isentropic efficiency model in the design operating area for the A270-L59 compressor.

\begin{tabular}{ccccc}
\hline Mathematical Model & $\boldsymbol{R}_{\mathbf{c}}^{\mathbf{2}(-)}$ & $\boldsymbol{M A P E}(\mathbf{\%})$ & $\mathbf{P E B}_{ \pm \mathbf{5} \%} \mathbf{( \% )}$ & $\boldsymbol{P E B}_{ \pm \mathbf{1 0}} \mathbf{( \% )}$ \\
\hline Karlson model & 0.9677 & 0.9670 & 98.9619 & 100 \\
Guan Cong model & 0.9927 & 0.4525 & 100 & 100 \\
Second-order Zeng Tao model & 0.8123 & 2.3583 & 90.6574 & 100 \\
First-order Zeng Tao model & 0.7821 & 2.7456 & 89.2734 & 100 \\
Hadef model & 0.9641 & 0.9561 & 98.2699 & 100 \\
Llamas model & 0.9777 & 0.7602 & 99.6540 & 100 \\
\hline
\end{tabular}

Table 10. Error evaluation results of each compressor isentropic efficiency model in the design operating area for the TCA88-25070 compressor.

\begin{tabular}{ccccc}
\hline Mathematical Model & $\boldsymbol{R}_{\mathbf{c}}^{\mathbf{2}(-)}$ & $\mathbf{M A P E}(\mathbf{\%})$ & $\mathbf{P E B}_{ \pm \mathbf{5} \%} \mathbf{( \% )}$ & $\boldsymbol{P E B}_{ \pm \mathbf{1 0} \%}(\mathbf{\%})$ \\
\hline Karlson model & 0.3938 & 1.6497 & 89.6552 & 100 \\
Guan Cong model & 0.9496 & 0.7067 & 100 & 100 \\
Second-order Zeng Tao model & 0.7408 & 1.6784 & 98.2759 & 100 \\
First-order Zeng Tao model & 0.8007 & 1.5128 & 100 & 100 \\
Hadef model & 0.8964 & 0.9805 & 98.2759 & 100 \\
Llamas model & 0.9102 & 0.8678 & 100 & 100 \\
\hline
\end{tabular}

By observing and analyzing the comparative and error evaluation results, it was found that in general, all the investigated models are able to characterize the changing trend of isentropic efficiency with the mass flow rate for both the A270-L59 and TCA55-25070 marine compressors. Meanwhile, satisfactory prediction accuracy was attained for all the models and compressors with a $P E B_{ \pm} 10 \%$ equal to $100 \%, P E B_{ \pm 5} \%$ greater than $89 \%$, and a MAPE less than $2.8 \%$.

Among these models, the Guan Cong model achieved the best prediction accuracy for both compressors, which is mainly attributed to its zonal modeling methodology and the correction procedure adopted, as introduced in Section 3.2.2. Although the predictive accuracy of both the Hadef model and the Llamas model were slightly inferior to the Guan Cong model, they were still satisfactory, with a MAPE of less than $1 \%$ and a $P E B_{ \pm 5} \%$ approaching $100 \%$ for both compressors, and this is attributed to the excellent parameterization results achieved with $R^{2}$ approaching one, and the strong physical fundamentals embedded in the two models. It should be noted that although the Llamas model can be regarded as an improved version of the Hadef model, the improvement level is not very evident for the two marine compressors investigated in this research, as can be observed in Tables 9 and 10. Despite the excellent parameterization results achieved for both the first- and second-order Zeng Tao models, with $R^{2}$ approaching one, their predictive accuracy was inferior compared to the Guan Cong, Hadef, and Llamas models with a MAPE around 1.6\% for the TCA55-25070 compressor, and around $2.5 \%$ for the A270-L59 compressor. Nevertheless, it should be noted that the predictive accuracy of the Zeng Tao model was still satisfactory if the requirement on predictive accuracy was not extremely high, because the $P E B_{ \pm 10 \%}$ of this model was still $100 \%$ and the $M A P E$ was between $1.5 \%$ and $2.8 \%$, for both compressors. In addition, the Zeng Tao model presents strong practicability in engineering practice, as it can be parameterized with a relatively small number of measured sample data. The predictive accuracy of the Karlson model dropped moderately when the modeling object switched from the A270-L59 to the TCA55-25070 compressor; in addition, the $R_{c}^{2}$ of this model was only 0.3939 for the TCA55-25070 compressor, and this is due to the fact that the number of model parameters existing in the Karlson model is too much with respect to the amount of measured sample data existing for this particular compressor ( 21 model parameters and 54 measured sample data). 


\subsection{Off-Design Operating Area}

\subsubsection{Low-Pressure Ratio (LR) Area}

Similar to the compressor mass flow rate model, the isentropic efficiency model is also required to extrapolate to the LR area. In general, only operating points with relatively high isentropic efficiency are measured and depicted in the compressor performance map, for instance, the lowest isentropic efficiency value in the performance map of A270-L59 marine compressor is as high as 65\%, and it is $74 \%$ for the TCA55-25070 compressor. Due to the absence of corresponding measured data points, it is difficult to study the LR area extrapolation ability for each isentropic efficiency model.

For the isentropic efficiency model, the compressor rotational speed, pressure ratio, and mass flow rate are generally selected as the input variables, and it should be noted that $\eta$ will be necessarily zero when $\Pi$ is equal to one according to its definition. Although the mass flow rate when $\Pi$ is equal to 1 is generally not provided in the performance map, its value $\dot{m}_{\Pi=1}$ can be estimated with the help of Leufvén and Llamas ellipse model that manifests superior LR area extrapolation ability. Based on the above analysis, the LR area extrapolation ability of each model can be investigated by comparing $\eta=0$ and the predicted isentropic efficiency $\eta_{\Pi=1}$ with $\Pi=1$ and corresponding $\dot{m}_{\Pi=1}$ as model input at each rotational speed condition.

Since the definition of isentropic efficiency is directly utilized to estimate the isentropic efficiency of the Zeng Tao, Hadef, and Llamas models, therefore the predicted isentropic efficiency will necessarily

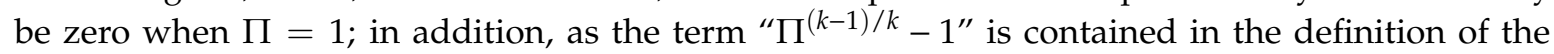
dimensionless torque coefficient $\Gamma$, as shown in Equation (24), a similar result would also be obtained by the Guan Cong model. As a result, only the LR area extrapolation ability of the Karlson model was required to be investigated. As observed in Tables 11 and 12, $\eta_{\Pi=1}$ predicted by the Karlson model deviated from $\eta=0$ significantly for both compressors, and some of the predicted values were even greater than one or negative, which violates the physical meaning of the isentropic efficiency. The inferior LR area extrapolation ability of the Karlson model is mainly due to its theoretical foundation, which is based on multiple regression and lacks strong physical fundamentals.

Table 11. Predicted isentropic efficiency value from the Karlson model when pressure ratio equals to 1 for the A270-L59 compressor.

\begin{tabular}{cccccccccc}
\hline$N$ (rpm) & 6800 & 7200 & 8400 & 9000 & 9600 & 10,200 & 10,800 & 11,400 & 12,000 \\
\hline$\eta_{\Pi=1}$ (-) & 0.3280 & 0.2774 & 0.2391 & 0.2040 & 0.1750 & 0.1576 & 0.1616 & 0.2015 & 0.2957 \\
\hline$N$ (rpm) & 12,600 & 13,200 & 13,800 & 14,400 & 15,000 & 15,600 & 16,200 & 16,800 & \\
\hline$\eta_{\Pi=1}(-)$ & 0.4618 & 0.7099 & 1.0371 & 1.4278 & 1.8592 & 2.3085 & 2.7572 & 3.1921 & \\
\hline
\end{tabular}

Table 12. Predicted isentropic efficiency value from the Karlson model when pressure ratio equals to 1 for TCA55-25070 compressor.

\begin{tabular}{cccccccccc}
\hline$U(\mathrm{~m} / \mathrm{s})$ & 250 & 300 & 350 & 400 & 450 & 475 & 500 & 525 & 550 \\
\hline$\eta_{\Pi=1}(-)$ & 0.1813 & 0.0018 & -0.012 & 0.8108 & 4.2292 & 7.9894 & 13.27 & 18.74 & 23.88 \\
\hline
\end{tabular}

\subsubsection{Low-Speed (LS) Area}

In Figures 13 and 14, the LS area extrapolation results of each model are compared with the A270-L59, and the TCA88-25070 compressors as modeling objects, respectively, and Tables 13 and 14 provide corresponding error evaluation results. 


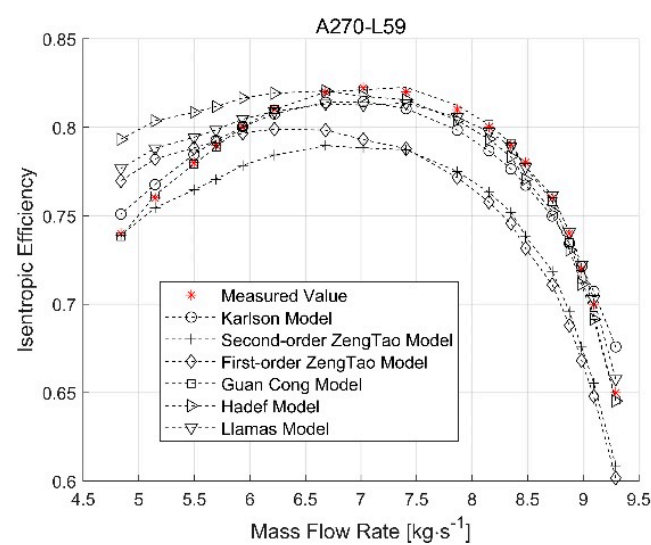

Figure 13. LS area extrapolation results for each compressor isentropic efficiency model for the A270-L59 marine compressor.

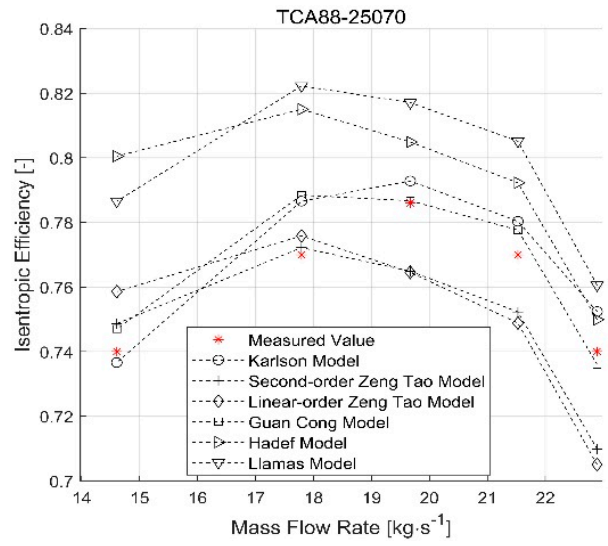

Figure 14. LS area extrapolation results for each compressor isentropic efficiency model for the TCA88-25070 marine compressor.

Table 13. Error evaluation results of LS area extrapolation results of each compressor isentropic efficiency model for the A270-L59 marine compressor.

\begin{tabular}{ccccccc}
\hline & $\begin{array}{c}\text { Karlson } \\
\text { Model }\end{array}$ & $\begin{array}{c}\text { Guan Cong } \\
\text { Model }\end{array}$ & $\begin{array}{c}\text { First-Order Zeng } \\
\text { Tao Model }\end{array}$ & $\begin{array}{c}\text { Second-Order } \\
\text { Zeng Tao Model }\end{array}$ & $\begin{array}{c}\text { Hadef } \\
\text { Model }\end{array}$ & $\begin{array}{c}\text { Llamas } \\
\text { Model }\end{array}$ \\
\hline MAPE $(\%)$ & 1.1266 & 0.3020 & 4.3071 & 4.0164 & 1.8218 & 1.0281 \\
\hline
\end{tabular}

Table 14. Error evaluation results of LS area extrapolation results of each compressor isentropic efficiency model for the TCA88-25070 marine compressor.

\begin{tabular}{ccccccc}
\hline & $\begin{array}{c}\text { Karlson } \\
\text { Model }\end{array}$ & $\begin{array}{c}\text { Guan Cong } \\
\text { Model }\end{array}$ & $\begin{array}{c}\text { First-Order Zeng } \\
\text { Tao Model }\end{array}$ & $\begin{array}{c}\text { Second-Order } \\
\text { Zeng Tao Model }\end{array}$ & $\begin{array}{c}\text { Hadef } \\
\text { Model }\end{array}$ & $\begin{array}{c}\text { Llamas } \\
\text { Model }\end{array}$ \\
\hline$M A P E(\%)$ & 1.2996 & 0.9929 & 2.6941 & 2.1021 & 4.1314 & 4.8751 \\
\hline
\end{tabular}

By observing the comparative and error evaluation results, it was found that in general, all the investigated models were able to achieve satisfactory LS area extrapolation accuracy with a MAPE lower than $4.5 \%$ for both compressors. Among these models, the Guan Cong model achieved the best predictive accuracy with a MAPE of only $0.3020 \%$ for the A270-L59 compressor, and $0.9929 \%$ for the TCA55-25070 compressor. Although the model parameters corresponding to the available lowest iso-speed line are adopted when extrapolating to LS area by the Guan Cong model, its superior extrapolation accuracy is mainly due to the fact that the change in rotational speed has only a minor influence on the fluid physical properties under low rotational speed conditions, and thus, similar changing trends between dimensionless torque coefficient and flow coefficient could be obtained. 


\subsubsection{High Speed (HS) Area}

In Figures 15 and 16, the LS area extrapolation results of each model are compared with the A270-L59, and the TCA88-25070 compressor as the modeling objects, respectively, and Tables 15 and 16 provide corresponding error evaluation results.

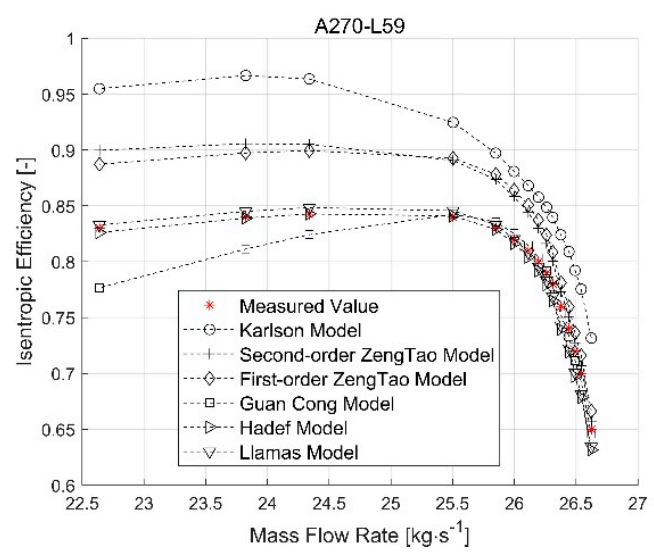

Figure 15. HS area extrapolation results for each compressor isentropic efficiency model for the A270-L59 marine compressor.

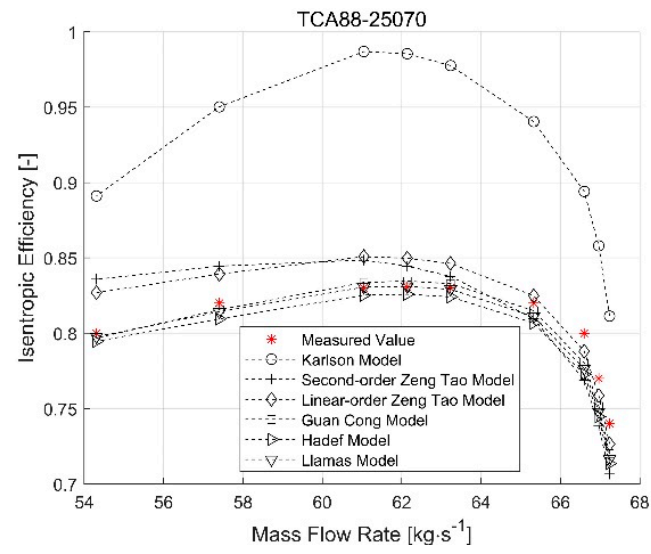

Figure 16. HS area extrapolation results for each compressor isentropic efficiency model for the TCA88-25070 marine compressor.

Table 15. Error evaluation results of HS area extrapolation results of each compressor isentropic efficiency model for the A270-L59 compressor.

\begin{tabular}{ccccccc}
\hline & $\begin{array}{c}\text { Karlson } \\
\text { Model }\end{array}$ & $\begin{array}{c}\text { Guan Cong } \\
\text { Model }\end{array}$ & $\begin{array}{c}\text { First-Order Zeng } \\
\text { Tao Model }\end{array}$ & $\begin{array}{c}\text { Second-Order } \\
\text { Zeng Tao Model }\end{array}$ & $\begin{array}{c}\text { Hadef } \\
\text { Model }\end{array}$ & $\begin{array}{c}\text { Llamas } \\
\text { Model }\end{array}$ \\
\hline$M A P E(\%)$ & 10.0404 & 1.3300 & 4.5335 & 3.9874 & 1.3989 & 1.2175 \\
\hline
\end{tabular}

Table 16. Error evaluation results of HS area extrapolation results of each compressor isentropic efficiency model for the TCA88-25070 compressor.

\begin{tabular}{ccccccc}
\hline & $\begin{array}{c}\text { Karlson } \\
\text { Model }\end{array}$ & $\begin{array}{c}\text { Guan Cong } \\
\text { Model }\end{array}$ & $\begin{array}{c}\text { First-Order Zeng } \\
\text { Tao Model }\end{array}$ & $\begin{array}{c}\text { Second-Order } \\
\text { Zeng Tao Model }\end{array}$ & $\begin{array}{c}\text { Hadef } \\
\text { Model }\end{array}$ & $\begin{array}{c}\text { Llamas } \\
\text { Model }\end{array}$ \\
\hline$M A P E(\%)$ & 14.4526 & 1.1521 & 1.9858 & 2.8896 & 1.7439 & 1.2565 \\
\hline
\end{tabular}

Among the five compressor isentropic efficiency models, the HS area extrapolation accuracy of the Karlson model was the lowest with a MAPE as high as $10.0404 \%$, and $14.4526 \%$ for the A270-L59 and the TCA55-25070 compressor, respectively, and this is mainly due to it lacking strong physical 
fundamentals. Although the HS area extrapolation accuracy of the Guan Cong model dropped slightly when compared to its LS area extrapolation accuracy, it still achieved the best predictive accuracy among these models in the HS area.

By observing the error evaluation results shown in Tables 9-16, it can be seen that for the Zeng Tao, Hadef and Llamas models, their predictive accuracy was relatively stable and satisfactory in both the design and off-design areas. This is the consequence of the relatively strong physical fundamentals embedded in these models.

\section{Summary and Discussion}

In this paper, a comprehensive applicable and comparative study of compressor mass flow rates and empirical isentropic efficiency models was carried out and performed on two marine compressors with different impeller outlet diameters, flow ranges, and rotational speed ranges. In general, consistent comparative analysis results were obtained for the two different compressors, and they are summarized as follows:

Compressor mass flow rate model:

- In general, In the design operating area, all of the investigated models were capable of characterizing the changing trend between mass flow rate and pressure ratio, among which the Guan Cong model achieved the best predictive accuracy, mainly owing to its zonal modeling methodology;

- Besides the design operating area, superior LS and LP extrapolation accuracy were also achieved by the Guan Cong model. However, it dropped significantly in the HS area for both compressors due to the considerable influence of rotational speed on the fluid physical properties under high-speed conditions;

- A satisfactory predictive result was achieved by both the Müller-I and -II models under low rotational speed conditions, which is mainly attributed to its theory foundation;

- The Leufvén and Llamas ellipse model was capable of describing the compressor choking phenomenon accurately and reasonably, including both the choking flow and choking pressure. In addition, the Guan Cong, Karlson-I, Karlson-II, and Kolmanovsky models could also describe the choking phenomenon, but they were inferior to the ellipse model;

- It is interesting to note that satisfactory predictive and extrapolation accuracy was achieved by both the Karlson-I and -II models for the A270-L59 compressor. However, the HS area extrapolation ability of the two models deteriorated significantly when the TCA88-25070 compressor was selected as the modeling object, which indicates that their HS area extrapolation ability is unstable and may vary with compressors.

Compressor isentropic efficiency model:

- When extrapolating to LP and HS area, the Karlson model manifested an undesirable extrapolation ability mainly due to its theoretical foundation, which is based on multiple regression and lacks strong physical fundamentals;

- In the design operating area, the predictive accuracy of the Guan Cong model was the highest, mainly owing to its zonal modeling methodology and the adopted correction procedure. Additionally, this model also had satisfactory LP, LS, and HS extrapolation abilities;

- Owing to the strong quadratic relationship existing between the power and speed coefficients, and the strong linear relationship between the logarithm of power and speed coefficients, the Zeng Tao model was very practical in engineering practice, as it can be parameterized readily with a relatively small number of sample data points.

- For the whole operating area, both the Hadef model and the Llamas model provided satisfactory predictive and extrapolation ability for both compressors investigated in this paper, and this is because that the theoretical foundation of the two models are based on basic physical laws (the Euler equation for turbomachinery and the laws of thermodynamics, for instance) and the 
definition of isentropic efficiency is directly utilized. In addition, the predictive and extrapolation ability of the Llamas model was not improved, although obviously, it is regarded as an improved version of the Hadef model.

It can be found from the applicable and comparative research that all the models investigated in this paper belong to the empirical model category, and this is mainly due to the fact that this type of model has a simpler structure and is easier to tune, and its predictive and extrapolation ability was satisfactory to some extent. Consequently, empirical compressor models are widely adopted in the study of performance, energy analysis and control- or diagnosis-oriented simulations. Nevertheless, due to relatively weaker physical fundamentals embedded in these empirical models, relative to their theoretical counterparts, satisfactory predictive and extrapolation ability cannot be guaranteed for an arbitrary compressor. For example, the LS area extrapolation accuracy of both the Karlson-I and -II models dropped significantly when the modeling object switched from the A270-L59 to the TCA88-25070 compressor, as shown in Section 5.2.2. Undoubtedly, the research carried out in this paper will be more meaningful if these empirical models are additionally compared with theoretical models in terms of simulation speed, predictive and extrapolation accuracy, and complexity in model tuning. However, a detailed compressor geometry dimension is always required for developing theoretical models, which is usually difficult to obtain for large-scale marine compressors including the two compressors investigated in this paper. Actually, the ultimate purpose of this paper was to find a compressor model that is most suitable for the modeling of marine two-stroke diesel engines used for marine engineering simulation, which has a relatively high requirement on both simulation speed and predictive accuracy, and it can be seen from the comparative analysis results that some of the empirical models satisfied this requirement very well.

Another point that should be noted is that none of these empirical compressor models appear to achieve satisfactory predictive and extrapolation accuracy in the whole operating area simultaneously. For instance, although extremely satisfactory LR area extrapolation accuracy was achieved by the Leufvén and Llamas ellipse mass flow rate model, its predictive ability in design operating area and extrapolation ability in LS and HS area was undesirable. Actually, this limitation can be broken with a zonal modeling approach, i.e., selecting the model with the best predictive accuracy for each operating area. For example, for the A270-L59 compressor, the design and LS operating area can be modeled by the Guan Cong model, LP area modeled by the ellipse model, and HS area modeled by the Karlson-II model. However, it should be noted that an appropriate blending algorithm is required to guarantee a smooth transition at the border between different operating areas. The authors will follow this path and construct a set of empirical models, which can achieve satisfactory predictive accuracy in the whole operating area.

Generally, for achieving satisfactory predictive results with these empirical models, a large number of measured sample data is required for model tuning and parameterization. For a small-scale automotive compressor, its performance map can be readily obtained from the manufacturers, or even be found on the internet. However, as large-scale marine compressor turbocharger manufacturers rarely provide its performance maps, it makes the model parameterization procedure very difficult. Nevertheless, the shop test report of the marine diesel engine usually provides compressor operating performance at several engine loading points, including rotational speed, pressure and temperature at the inlet and outlet, and sometimes mass flow rate. Therefore, it will be very practical to develop a kind of compressor model that can be parameterized only with these measured performance data provided by the shop test report. An approximate methodology, following this idea proposed by Theotokatos [37], is often adopted by authors when developing simulation models for marine diesel engines in cases where the compressor performance map is lacking. This method is based on the fact that the engine is loaded according to the propeller law in a marine propulsion plant, and the operating points of the compressor lie on a single curve on the compressor map under steady-state conditions; in addition, this curve is close to the optimum compressor efficiency parabola owing to the fine matching between the turbocharger and engine. Consequently, the compressor pressure ratio 
and isentropic efficiency can be modeled as second-order polynomial functions of the turbocharger rotational speed with only three model parameters at most, which can be then parameterized readily with the compressor operating performance at several loading points. Similarly, as strong quadratic relationship exists between the power and speed coefficient and strong linear relationship between the logarithm of power and speed coefficient in the Zeng Tao isentropic efficiency model [36], the R-square of fitting results of which was more than 0.99 for both the A270-L59 and the TCA88-25070 marine compressors. Thus, this model can be parameterized readily only with the compressor performance information at the five to six operating points provided by shop test reports. In general, with fewer model parameters existing in the empirical models, the model parameterization procedure becomes much easier. However, it should be noted that the parameterization result is expected to be more reliable with more measured sample data available.

\section{Conclusions}

A comprehensive applicable and comparative study of compressor mass flow rate and isentropic efficiency empirical models was carried out on two large-scale marine compressors. Based on the comparative analysis results obtained in this research, several guidelines are summarized, which can be followed when developing simulation models for both compressor mass flow rate and isentropic efficiency:

- Different types of empirical compressor models manifest different predictive accuracy and extrapolation ability in different operating areas; therefore, it is necessary to select the most suitable modeling methodology for the particular area concerned;

- The zonal modeling approach proposed and implemented by Guan et al. [34] is able to effectively improve the predictive accuracy for both compressor mass flow rate and isentropic efficiency models. However, its LS and HS area extrapolation strategy requires special attention as the change in compressor rotational speed has influences on fluid physical properties especially compressibility under high-speed conditions;

- To ensure robust extrapolation ability and reliable extrapolation results, it is indispensable to enhance the physical fundamentals of the developed compressor models;

- When developing compressor models, it is necessary to pay special attention to their sensitivity to the errors in model parameters, and this is due to the fact that if the sensitivity is high, satisfactory predictive accuracy cannot be guaranteed even though the parameterization result is satisfactory, with a high value of $R_{\mathrm{c}}^{2}$ for instance;

- Since the mass flow rate is normally selected as the input variable for the isentropic efficiency model, the predictive errors of the mass flow rate model will propagate to the isentropic efficiency model. So, it will be beneficial to parameterize the mass flow rate and isentropic efficiency model simultaneously with an appropriate parameterization algorithm in order to achieve satisfactory predictive accuracy in the three dimensions, including the pressure ratio, mass flow rate, and isentropic efficiency.

Both the A270-L59 and TCA88-25070 compressors are very representative of the air boosting of large two-stroke marine diesel engines, and consistent comparative analysis results were obtained for the two different compressors. Thus, the research conducted in this paper is believed to be very helpful for researchers who are interested in the dynamic simulation study of large two-stroke marine diesel engines to select the best mathematical models for their application and research.

Author Contributions: H.S. contributed to the parameterization of mathematical models, analysis of simulation results, and the writing of the manuscript. C.Z. provided language help and some technical support. J.Z. provided research conditions, team support, and financial support. B.Y. and B.J. gave some useful suggestions. All authors have read and agreed to the published version of the manuscript.

Funding: This work was supported by the National Natural Science Foundation of China, grant number 51479017, and the Fundamental Research Funds for the Central Universities, grant number 3132019315. 
Conflicts of Interest: The authors declare no conflict of interest.

\section{References}

1. Song, K.; Upadhyay, D.; Xie, H. A physics-based turbocharger model for automotive diesel engine control applications. Proc. Inst. Mech. Eng. Part D J. Automob. Eng. 2019, 233, 1667-1686. [CrossRef]

2. Taburri, M.; Chiara, F.; Canova, M.; Wang, Y.Y. A model-based methodology to predict the compressor behaviour for the simulation of turbocharged engines. Proc. Inst. Mech. Eng. Part D J. Automob. Eng. 2012, 226, 560-574. [CrossRef]

3. Leufvén, O.; Eriksson, L. A surge and choke capable compressor flow model-validation and extrapolation capability. Control Eng. Pract. 2013, 21, 1871-1883. [CrossRef]

4. Tu, H. Investigation on the Modeling and Speed Control of Marine Diesel Engine Propulsion System. Ph.D. Dissertation, Wuhan University of Technology, Wuhan, China, 2015.

5. Malkhede, D.N.; Seth, B.; Dhariwal, H.C. Mean value model and control of a marine turbocharged diesel engine. In Proceedings of the Powertrain and Fluid Systems Conference and Exhibition, San Antonio, TX, USA, 24-27 September 2005.

6. Karlson, A.T. On Modeling of a Ship Propulsion System for Control Purposes. Master's Dissertation, Norwegian University of Science and Technology, Trondheim, Norway, 2012.

7. Hansen, J.M.; Zander, C.G.; Pedersen, N.; Blanke, M.; Vejlgaard-Laursen, M. Modeling for control of exhaust gas recirculation on large diesel engines. In Proceedings of the 9th IFAC Conference on Control Applications in Marine Systems, Osaka, Japan, 17-20 September 2013.

8. Sun, J.B.; Guo, C.; Wei, H.J.; Yu, H.L.; Huang, J.L. Calculation and characteristic analysis on turbocharger surging of marine diesel engine. Dalian Haishi Daxue Xuebao 2008, 34, 28-31, 36. [CrossRef]

9. Tu, H.; Chen, H. Modeling of compressor characteristics using Kriging method. Neiranj Xuebao 2014, 32, 377-383. [CrossRef]

10. Wang, H.Y.; Hao, J.W.; Zhang, X.S.; Liu, C. Thermodynamic model of turbocharger and dynamic simulation of diesel engine. Neiranji Gongcheng 2017, 38, 128-134. [CrossRef]

11. Sakellaridis, N.F.; Rapotasios, S.I.; Antonopoulos, A.K.; Mavropoulos, G.C.; Hountalas, D.T. Development and validation of a new turbocharger simulation methodology for marine two stroke diesel engine modeling and diagnostic applications. Energy 2015, 91, 952-966. [CrossRef]

12. Fang, X.D.; Chen, W.W.; Zhou, Z.R.; Xu, Y. Empirical models for efficiency and mass flow rate of centrifugal compressors. Int. J. Refrig. 2014, 41, 190-199. [CrossRef]

13. Yang, B.; Fang, X.D.; Zhang, L.S.; Zhuang, F.T.; Bi, M.H.; Chen, C.; Li, G.; Wang, X. Applicability of empirical models of isentropic efficiency and mass flow rate of dynamic compressors to jet engines. Prog. Aerosp. Sci. 2019, 106, 32-42. [CrossRef]

14. Eriksson, L.; Nezhadali, V.; Andersson, C. Compressor flow extrapolation and library design for the modelica vehicle propulsion library-vehprolib. In Proceedings of the SAE 2016 World Congress and Exhibition, Detroit, MI, USA, 12-14 April 2016.

15. McMullen, R.; Pino, Y. Conditioning turbocharger map data for use in engine performance simulation. $S A E$ Int. J. Engines 2018, 11, 491-507. [CrossRef]

16. Llamas, X.; Eriksson, L. Parameterizing compact and extensive compressor models using orthogonal distance minimization. J. Eng. Gas Turbines Power 2016, 139, 012601. [CrossRef]

17. Dinescu, D.C.; Tazerout, M. Mean value modeling of a variable nozzle turbocharger (VNT). UPB Sci. Bull. Ser. D 2010, 72, 109-116.

18. Jensen, J.P.; Kristensen, A.F.; Sorenson, S.C.; Houbak, N.; Hendricks, E. Mean value modeling of a small turbocharged diesel engine. In Proceedings of the International Congress and Exposition, Detroit, MI, USA, 25 February-1 March 1991.

19. Hadef, J.E.; Colin, G.; Chamaillard, Y.; Talon, V. Physical-based algorithms for interpolation and extrapolation of turbocharger data maps. SAE Int. J. Engines 2012, 5, 363-378. [CrossRef]

20. Martin, G.; Talon, V.; Higelin, P.; Charlet, A.; Cailol, C. Implementing turbomachinery physics into data map-based turbocharger models. SAE Int. J. Engines 2009, 2, 211-229. [CrossRef]

21. Wang, H.Y. Modeling and Simulation of Large-Scale Low-Speed Marine Diesel Engine. Ph.D. Dissertation, Dalian Maritime University, Dalian, China, 2006. 
22. Kao, M.; Moskwa, J.J. Turbocharged diesel engine modeling for nonlinear engine control and state estimation. ASME J. Dyn. Syst. Meas. Control Trans. 1995, 117, 20-30. [CrossRef]

23. Moraal, P.; Kolmanovsky, I. Turbocharger modeling for automotive control applications. In Proceedings of the International Congress and Exposition, Detroit, MI, USA, 1-4 March 1999.

24. Ghorbanian, K.; Gholamrezaei, M. An artificial neural network approach to compressor prediction. Appl. Energy 2009, 86, 1210-1221. [CrossRef]

25. Gholamrezaei, M.; Ghorbanian, K. Compressor map generation using a feed-forward neural network and rig data. Proc. Inst. Mech. Eng. Part A J. Power Eng. 2010, 224, 97-108. [CrossRef]

26. Stricker, K.; Kocher, L.; Koeberlein, E.; Alstine, D.G.V.; Shaver, G.M. Turbocharger map reduction for control oriented modeling. ASME J. Dyn. Syst. Meas. Control Trans. 2014, 136, 041008. [CrossRef]

27. Canova, M.; Naddeo, M.; Liu, Y.X.; Zhou, J.Q.; Wang, Y.Y. A scalable modeling approach for the simulation and design optimization of automotive turbochargers. SAE Int. J. Engines 2015, 8, 1616-1628. [CrossRef]

28. Llamas, X.; Eriksson, L. Control-oriented compressor model with adiabatic efficiency extrapolation. SAE Int. J. Engines 2017, 10, 1903-1916. [CrossRef]

29. Leufvén, O.; Eriksson, L. Measurement, analysis and modeling of a centrifugal compressor flow for low pressure. Int. J. Engine Res. 2016, 17, 153-168. [CrossRef]

30. Nakhjiri, M.; Pelz, P.; Matyschok, B.; Daubler, L.; Horn, A. Physical modeling of automotive turbocharger compressor: Analytical approach and validation. In Proceedings of the SAE 2011 Commercial Vehicle Engineering Congress, Rosemont, IL, USA, 13-14 September 2011.

31. Swain, E. Improving a one-dimensional centrifugal compressor performance prediction method. Proc. Inst. Mech. Eng. Part A J. Power Eng. 2005, 219, 653-660. [CrossRef]

32. Jiang, W.; Khan, J.; Dougal, R.A. Dynamic centrifugal compressor model for system simulation. J. Power Sources 2006, 158, 1333-1343. [CrossRef]

33. Yin, J.G.; Su, T.X.; Guan, Z.W.; Chu, Q.H.; Meng, C.J.; Jia, L.; Wang, J.; Zhang, Y.G. Modeling and validation of a diesel engine with turbocharger for hardware-in-the-loop applications. Energies 2017, 10, 685. [CrossRef]

34. Guan, C.; Theotokatos, G.; Zhou, P.; Chen, H. Computational investigation of a large containership propulsion engine operation at slow steaming conditions. Appl. Energy 2014, 130, 370-383. [CrossRef]

35. Müller, M.; Hendricks, E.; Sorenson, S.C. Mean value modeling of turbocharged spark ignition engines. In Proceedings of the 1998 SAE International Congress and Exposition, Detroit, MI, USA, 23-26 February 1998.

36. Zeng, T.; Upadhyay, D.; Zhu, G. A reduced complexity model for the compressor power of an automotive turbocharger. J. Dyn. Syst. Meas. Control Trans. ASME 2018, 140, 061018. [CrossRef]

37. Theotokatos, G. On the cycle mean value modelling of a large two-stroke marine diesel engine. Proc. Inst. Mech. Eng. Part M J. Eng. Marit. Environ. 2010, 224, 193-205. [CrossRef] 